\title{
Actualización taxonómica de las algas pardas (Phaeophyceae, Ochrophyta) marinas bentónicas del Atlántico mexicano
}

\author{
Taxonomic update of the benthic marine brown algae \\ (Phaeophyceae, Ochrophyta) from the Mexican Atlantic
} Acta Botanica Mexicana

\author{
Annie May Ek García-Garcíal(D) Ernesto Cabrera-Becerril2(D) María Luisa Núñez-Resendiz2,3(D, \\ Kurt M. Dreckmann²(1) Abel Sentíes²D
}

\section{Resumen:}

Antecedentes y Objetivos: Con excepción de las compilaciones de 2020 para algas rojas y la ficoflora en México, desde los catálogos previos al 2001, no se han realizado otros trabajos que involucren la revisión y actualización del estatus de las algas pardas en el Atlántico mexicano. El objetivo de este estudio fue integrar un listado de algas pardas del Golfo de México y el Caribe mexicano en el que se consideren todas las actualizaciones taxonómicas de 2001 a la fecha.

Métodos: Se consultó la literatura especializada para la región publicada de 2001 a septiembre de 2021 y la base de datos del Herbario UAMIZ para la integración de un listado ficoflorístico con datos de distribución. La sinonimia y el estatus nomenclatural de cada nombre, así como el sistema de clasificación utilizado, fueron revisados en AlgaeBase.

Resultados clave: Se obtuvo un listado actualizado con 97 especies y tres categorías infraespecíficas de algas pardas, distribuidas en nueve órdenes, 12 familias y 39 géneros. Del total, del 2001 al presente, 41 corresponden con adiciones a la flora, 24 son nombres actualizados, 10 son registros antiguos que no han vuelto a ser mencionados y 25 siguen siendo citados.

Conclusiones: Es evidente la necesidad de realizar estudios florísticos combinados con enfoques moleculares, que proporcionen un respaldo para un conocimiento más certero e integral de los recursos algales mexicanos y que posibiliten la incursión en estudios ecológicos o biogeográficos que permitan establecer propuestas de manejo sustentable o conservación.

Palabras clave: distribución, diversidad, listado ficoflorístico, macroalgas marinas, nomenclatura.

\section{Abstract:}

Background and Aims: Except for the 2020 compilations for red algae and the phycoflora in Mexico, since the catalogs prior to 2001 , no other studies have been carried out that involve the review and update of the status of brown algae in the Mexican Atlantic. The aim of this study was to integrate a list of brown algae from the Gulf of Mexico and the Mexican Caribbean in which all taxonomic updates from 2001 to date are considered.

Methods: The specialized literature for the region published from 2001 to September 2021 and the database of the Herbarium UAMIZ were consulted for the integration of a phycofloristic list with distribution data. The synonymy and nomenclatural status of each name, as well as the classification system used, were reviewed in AlgaeBase.

Key results: An updated list was obtained with 97 species and three infraspecific categories of brown algae, distributed in nine orders, 12 families and 39 genera. Of the total, from 2001 to the present, 41 correspond to additions to the flora, 24 are updated names, 10 are old records that have not been mentioned again and 25 continue to be recorded.

Conclusions: The need to carry out floristic studies combined with molecular approaches It is evident, providing support for a more accurate and comprehensive knowledge of Mexican algal resources and making possible the incursion into ecological or biogeographic studies that allow the establishment of proposals for sustainable management or conservation.

Key words: distribution, diversity, floristics, marine macroalgae, nomenclature.

${ }^{1}$ Universidad Nacional Autónoma de México, Facultad de Ciencias, Coyoacán, Apdo. postal 70-474, 04510 Cd. Mx., México.

${ }^{2}$ Universidad Autónoma Metropolitana-Iztapalapa, Departamento de Hidrobiología, Apdo. postal 55-535, 09340 Cd. Mx., México.

${ }^{3}$ Autor para la correspondencia: mlnr@ciencias.unam.mx
Recibido: 10 de septiembre de 2021.

Revisado: 18 de septiembre de 2021

Aceptado por Rosario Redonda-Martínez: 11 de noviembre de 2021.

Publicado Primero en línea: 18 de noviembre de 2021. Publicado: Acta Botanica Mexicana 128(2021).

cc) (1) Este es un artículo de acceso abierto By bajo la licencia Creative Commons 4.0 Atribución-No Comercial (CC BY-NC 4.0 Internacional).
Citar como: García-García, A. M. E., E. Cabrera-Becerril, M. L. Núñez-Resendiz, K. M. Dreckmann y A. Sentíes. 2021. Actualización taxonómica de las algas pardas (Phaeophyceae, Ochrophyta) marinas bentónicas del Atlántico mexicano. Acta Botanica Mexicana 128: e1968. DOI: https://doi.org/10.21829/ abm128.2021.1968

e-ISSN: 2448-7589 


\section{Introducción}

A lo largo de 4374 km de extensión aproximada, tanto en línea de costa $(3294 \mathrm{~km})$ como en bordes insulares (1080 km), que comprenden el litoral del Golfo de México y el Caribe mexicano (Pedroche y Sentíes, 2003), se distribuye una amplia heterogeneidad de ambientes propicios para el establecimiento de macroalgas marinas (Pedroche et al., 2009; Sentíes y Dreckmann, 2011). De este grupo destacan las algas pardas, tanto por sus características morfológicas particulares que las asemejan estructuralmente, aunque no filogenéticamente a las plantas embriofitas (Adl et al., 2012), como por su abundancia en las costas en términos de biomasa (Dreckmann y Sentíes, 2013). Actualmente se conocen 2071 especies marinas con distribución mundial, tanto en ambientes templados, donde alcanzan tallas de hasta 60 m en longitud (Graham y Wilcox, 2000), como en ambientes tropicales, donde sus tallas son más pequeñas y varían entre 0.5 y $30 \mathrm{~cm}$ de longitud, pero son más diversas (Guiry y Guiry, 2021). Particularmente, en el Atlántico mexicano han sido registradas cerca de 100 especies distribuidas en nueve órdenes y 12 familias (Pedroche y Sentíes, 2020), lo que representa $5 \%$ de la diversidad del grupo en un contexto mundial.

Diversos estudios de índole florístico han permitido conocer la composición actual de las algas pardas en la región; los principales se encuentran sintetizados en dos grandes obras que reúnen los registros y la información relevante de estas especies: el catálogo de Dreckmann (1998) y el de Ortega et al. (2001). En conjunto, los listados florísticos permiten realizar estudios integrales de tipo ecológico, biogeográfico o taxonómico, entre los que se incluyen trabajos de determinación de riqueza y composición florística (Mateo-Cid et al., 2006; 2013a,b; Sentíes y Dreckmann, 2011, 2013; Tapia-Silva et al., 2015; Mendoza-González et al., 2017; Pérez-Jiménez et al., 2020), afinidad florística (Pedroche y Sentíes, 2020), determinación de patrones de distribución de taxones o localización de áreas de concentración de biota, áreas de endemismo o realización de propuestas de conservación (Vilchis et al., 2018).

Con la incorporación de marcadores moleculares al estudio de la diversidad de algas pardas, la taxonomía del grupo se ha modificado en todos los niveles jerárquicos. A pesar de que en México se conoce muy poco sobre la diver- sidad de este grupo, a diferencia de las algas rojas que han sido ampliamente estudiadas en la región (García-García et al., 2020), actualmente existen numerosos cambios nomenclaturales, principalmente al interior de muchos de los órdenes y familias que incluyen a las especies registradas para el Atlántico mexicano. Por ejemplo, muchos nombres han sido circunscritos a otros géneros (De Clerck et al., 2006; Lim et al., 2007; León-Álvarez et al., 2014a; Wynne y de Castro Nunes, 2021), algunos cayeron en desuso (Lozano-Orozco et al., 2014), otros se han detectado como erróneamente aplicados (Mattio y Payri, 2009; Díaz-Martínez et al., 2016), unos más han sido reestablecidos a un género (Berov et al., 2015; Boisset et al., 2016), muchas especies han sido descritas a partir de complejos crípticos (Boo et al., 2011; Lozano-Orozco et al., 2015; 2016) y otras se han reducido a sinonimia (León-Álvarez et al., 2014b, 2017, González-Nieto et al., 2020).

Desde las contribuciones de Dreckmann (1998) y Ortega et al. (2001), no se han realizado otros trabajos compilatorios en el Atlántico mexicano que incorporen las modificaciones y actualizaciones taxonómicas anteriormente referidas para la flora de algas pardas en la región. Únicamente se cuenta con el listado actualizado de algas rojas de García-García et al. (2020) y la revisión ficoflorística y de la diversidad mexicana de Pedroche y Sentíes (2020). Considerando que los listados florísticos son el primer acercamiento al conocimiento de la diversidad de cualquier región determinada, es importante contar con un compendio actualizado de todos los trabajos que han introducido cambios a la sistemática de las algas pardas. Por lo anterior, la presente contribución tiene como objetivo principal integrar un listado de algas pardas del Golfo de México y el Caribe mexicano, en el que se consideren todas las actualizaciones taxonómicas realizadas de 2001 a septiembre de 2021.

\section{Materiales y Métodos}

\section{Área de estudio}

El Océano Atlántico es el segundo más extenso de la Tierra y comprende aproximadamente $20 \%$ de su superficie. El Golfo de México y el Caribe mexicano forman parte de este océano; entre ambos constituyen una extensión aproximada de 4374 km entre línea de costa y bordes insulares a 
los que nos referimos como Atlántico mexicano (Pedroche y Sentíes, 2003), nuestra área de estudio (Fig. 1).

En el Golfo de México se distingue la región norte, conformada por los estados de Tamaulipas y Veracruz, donde las corrientes son más intensas que en el sur y existe una amplia superficie de sustrato rocoso, por lo que la mayoría de las algas que se distribuyen en la zona son de ambientes litorales (Vilchis et al., 2018). Por otro lado, la región sur conformada por los estados de Tabasco y Campeche presenta corrientes menos intensas, en comparación con la zona norte, y grandes extensiones arenosas con poca superficie rocosa y considerables aportes de agua dulce provenientes de los principales sistemas lagunares, por lo que la mayor parte de la ficoflora en la región corresponde con ambientes insulares o estuarinos; muy pocos son litorales
(Sentíes y Dreckmann, 2013). La dinámica oceanográfica del Golfo de México tiene una fuerte influencia de la Corriente de Yucatán: al entrar al Golfo esta corriente se llama Corriente del Lazo, la cual penetra hacia el Norte, luego gira al Este, posteriormente al Sur y finalmente al Este, saliendo por el Estrecho de Florida y pasa después a integrarse a la Corriente del Golfo, que es la corriente más caudalosa del Océano Atlántico Norte (Vilchis et al., 2018). Las corrientes de Yucatán y del Lazo son parte del giro subtropical del Atlántico Norte, que es generado por los Vientos Alisios (Abascal et al., 2003).

El Caribe mexicano es la región más sureña del Atlántico mexicano y está conformada por los estados de Yucatán y Quintana Roo. Aquí es donde predominan las corrientes suaves debido a las grandes extensiones arenosas

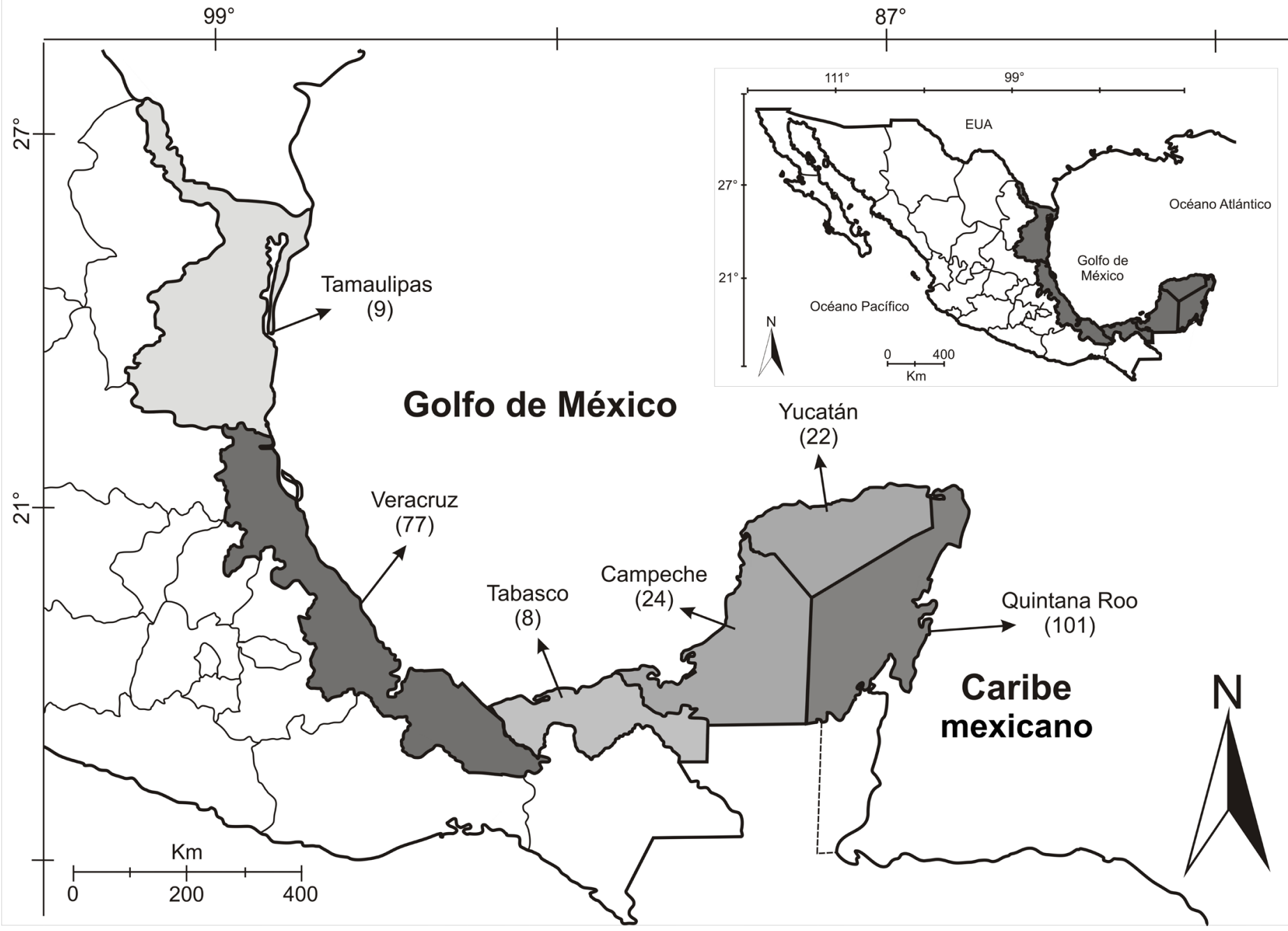

Figura 1: Mapa del Golfo de México y Caribe mexicano mostrando el total de localidades por estado que contienen registros de algas pardas (ver datos de distribución por especie en el listado florístico) en el Atlántico mexicano, indicados en paréntesis. 
previas al talud continental y a los pocos sustratos rocosos para el establecimiento de comunidades litorales, por lo que en esta región predomina la flora insular (Dreckmann y Sentíes, 2013). Campeche, Yucatán y Quintana Roo conforman la región emergida de la Península de Yucatán, la cual separa al Golfo de México y Caribe mexicano en dos regiones oceánicas y define sus características fisiográficas y patrones de corriente tan particulares.

\section{Revisión bibliográfica y de herbario}

La información de las especies de algas pardas registradas en el Golfo de México y en el Caribe mexicano se obtuvo de la revisión de literatura publicada para la región de 2001 a septiembre de 2021 (Cuadro 1), aunque también se incluyeron algunas otras publicaciones que no fueron consideradas en las revisiones anteriores (Dreckmann, 1998; Ortega et al., 2001). Adicionalmente, dicha revisión fue comple-

Cuadro 1: Publicaciones consultadas para la actualización florística de algas partas registradas en México, por estado: Tamaulipas (Tamps), Veracruz (Ver), Tabasco (Tabs), Campeche (Camp), Yucatán (Yuc) y Quintana Roo (QRoo). Las publicaciones previas a 2001 referidas en esta tabla no fueron consideradas previamente en Ortega et al., 2001. Con asterisco se indican las publicaciones en que se describen nuevas especies.

\begin{tabular}{|c|c|}
\hline Referencia & Estado \\
\hline Mateo-Cid y Mendoza-González, 1991 & QRoo \\
\hline Collado-Vides et al., 1995 & QRoo \\
\hline Ortega, 1995 & Camp \\
\hline Dreckmann et al., 1996 & QRoo, Yuc \\
\hline Mateo-Cid et al., 1996 & Ver \\
\hline Díaz-Martín y Espinoza-Ávalos, 2000 & Camp, Yuc, QRoo \\
\hline Mendoza-González y Mateo-Cid, 2000 & Ver, Yuc, QRoo \\
\hline Ortegón-Aznar et al., 2001 & Yuc \\
\hline Callejas Jiménez et al., 2005 & Camp \\
\hline Mendoza-González y Mateo-Cid, 2005 & Tamps, Ver, Camp, Yuc, QRoo \\
\hline Mateo-Cid et al., 2006 & QRoo \\
\hline Quan-Young et al., 2006 & QRoo \\
\hline González-Gándara et al., 2007 & Ver \\
\hline Mendoza-González et al., 2007 & QRoo \\
\hline Sánchez-Molina et al., 2007 & Yuc \\
\hline Aguilar-Rosas et al., 2008 & Ver, Camp, Yuc, QRoo \\
\hline Cetz-Navarro et al., 2008 & QRoo \\
\hline Godínez-Ortega et al., 2009 & Ver \\
\hline Ortegón-Aznar et al., 2009 & QRoo \\
\hline Ávila-Ortiz et al., 2011 & Ver, Camp, Yuc, QRoo \\
\hline Ramírez Rodríguez y Blanco Pérez, 2011 & Ver \\
\hline Robinson et al., 2012 & Ver \\
\hline Mateo-Cid et al., 2013a & Camp \\
\hline Mateo-Cid et al., 2013b & Camp \\
\hline Mendoza-González et al., 2013 & Camp \\
\hline Sentíes y Dreckmann, 2013 & Tabs \\
\hline González-Reséndiz et al., 2014 & Ver \\
\hline Valadez-Cruz et al., 2014 & QRoo \\
\hline León-Álvarez et al., 2014a & Ver \\
\hline *Lozano-Orozco et al., 2014 & Ver \\
\hline *Lozano-Orozco et al., 2015 & QRoo \\
\hline
\end{tabular}


Cuadro 1. Continuación.

\begin{tabular}{ll}
\hline Referencia & Estado \\
\hline *Lozano-Orozco et al., 2016 & Ver \\
*Díaz-Martínez et al., 2016 & Tamps, Ver, Camp, Yuc, QRoo \\
Mendoza-González et al., 2016 & Camp, Yuc, QRoo \\
Luna-Ortega y de la Cruz-Francisco, 2017 & Ver, QRoo \\
De la Cruz-Francisco et al., 2017 & Ver \\
Mendoza-González et al., 2017 & Tamps, Tabs \\
Nava-Olvera et al., 2017 & Ver, QRoo \\
Godínez-Ortega et al., 2018 & Ver, QRoo \\
Godínez-Ortega et al., 2019 & Ver \\
Landa-Cansigno et al., 2019 & Ver, Tabs \\
Mateo-Cid et al., 2019 & QRoo \\
Pérez-Jiménez et al., 2020 & Tabs \\
*González-Nieto et al., 2020 & Ver, QRoo \\
Colección Ficológica UAMIZ & Ver, Tabs, Camp, QRoo \\
\hline
\end{tabular}

mentada con los registros de la base de datos del Herbario UAMIZ de la Universidad Autónoma Metropolitana (acrónimo según Thiers, 2021), la cual no se encuentra en línea, por lo que los ejemplares se consultaron directamente en la colección. La sinonimia, el estatus nomenclatural de cada nombre registrado y su clasificación actual fueron revisados en la base de datos AlgaeBase (Guiry y Guiry, 2021).

\section{Resultados}

Se presenta el listado florístico actualizado de algas pardas con distribución en 241 localidades del Golfo de México (118) y Caribe mexicano (123) (Fig. 1), el cual incorpora 97 especies y tres categorías infraespecíficas distribuidas en nueve ordenes, 12 familias y 39 géneros. Del total de taxones registrados en la literatura existente de 2001 a septiembre de 2021, 41 corresponden con adiciones a la flora, 24 son nombres actualizados que habían sido registrados previamente en el área bajo algún sinónimo taxonómico, 10 son registros que no han vuelto a mencionarse en los listados florísticos (señalados con un asterisco) y 25 continúan registrándose. Para cada especie se indica su estatus nomenclatural, la sinonimia bajo la que fueron registradas previamente y los datos de distribución por estado y ambiente (litoral, insular o estuarino), así como las referencias a las obras en que se citan. Adicionalmente se incluyen comentarios taxonómicos en los casos en que se requiere precisar cambios taxonómicos importantes.

\section{Listado florístico}

Asterocladales

\section{Asterocladaceae}

Asterocladon rhodochortonoides (Børgesen) S. Uwai, C. Nagasato, T. Motomura \& K. Kogame.

三 Asteronema rhodochortonoides (Børgesen) D.G. Müller \& Parodi Sin.

E Ectocarpus rhodochortonoides Børgesen.

三 Herponema rhodochortonoides (Børgesen) Levring.

Distribución: Tamaulipas: litoral (como $H$. rhodochortonoides, Ortega et al., 2001); Tabasco: litoral (MendozaGonzález et al., 2017); Campeche: estuarino, litoral (como Asteronema rhodochortonoides, Ortega et al., 2001); Yucatán: insular (como Asteronema rhodochortonoides, Ortega et al., 2001); Quintana Roo: estuarino, litoral, insular (como E. rhodochortonoides, Collado-Vides et al., 1995; Dreckmann et al., 1996; como Asterocladon rhodochortonoides, Díaz-Martín y Espinoza-Ávalos, 2000; Ortega et al., 2001; Mendoza-González et al., 2016).

Comentarios taxonómicos: en Tamaulipas fue registrada como $H$. rhodochortonoides, actualmente considerada si- 
nónimo homotípico de Asteronema rhodochortonoides y a su vez de Asterocladon rhodochortonoides.

\section{Dictyotales}

\section{Dictyotaceae}

Canistrocarpus cervicornis (Kützing) De Paula \& De Clerck.

इ Dictyota cervicornis Kützing.

= Dictyota bartayresiana var. divaricata J. Agardh.

= Dictyota indica Sonder ex Kützing.

Distribución: Veracruz: estuarino, litoral, insular (Luna Ortega y de la Cruz-Francisco, 2017; Nava-Olvera et al., 2017; Landa-Cansigno et al., 2019; De la Cruz-Francisco et al., 2017; como D. bartayresiana var. divaricata, Mateo-Cid et al., 1996; como D. cervicornis, Sánchez-Rodríguez, 1980; Ortega et al., 2001; Ramírez Rodríguez y Blanco Pérez, 2011; Robinson et al., 2012; L. E. Mateo-Cid s.n. (UAMIZ-1288), J. Lozano-Orozco s.n. (UAMIZ 1289, UAMIZ 1290, UAMIZ 1291, UAMIZ 1292); Tabasco: estuarino (Pérez-Jiménez et al., 2020); Campeche: estuarino, litoral, insular (como D. cervicornis, Díaz-Martín y Espinoza-Ávalos, 2000; Ortega et al., 2001; Callejas Jiménez et al., 2005; Mateo-Cid et al., 2013a, b; Mendoza-González et al., 2013; MendozaGonzález et al., 2016; como D. cervicornis, M. Callejas s.n. (UAMIZ 932, UAMIZ 950)); Yucatán: litoral, insular (Mendoza-González et al., 2016; como D. cervicornis, Díaz-Martín y Espinoza-Ávalos, 2000; como D. indica y D. cervicornis, Ortega et al., 2001; Sánchez-Molina et al., 2007); Quintana Roo: estuarino, litoral, insular (Mendoza-González et al., 2016; Mateo-Cid et al., 2019; como D. cervicornis, MateoCid y Mendoza-González, 1991; Dreckmann et al., 1996; Díaz-Martín y Espinoza-Ávalos, 2000; Ortega et al., 2001; Quan-Young et al., 2006; Mateo-Cid et al., 2006; MendozaGonzález et al., 2007; Valadez-Cruz et al., 2014, como D. cervicornis, A. Senties y L. Morquecho s.n. (UAMIZ 539, UAMIZ 552), K. M. Dreckmann y A. Sentíes s.n. (UAMIZ 594), A. Senties s.n. (UAMIZ 659)).

Comentarios taxonómicos: especie ampliamente registrada en el Atlántico mexicano como D. cervicornis, transferida por De Clerck et al. (2006) al género Canistrocarpus De Paula \& De Clerck, descrito por los mismos autores a partir de secuencias de $r b c L$ y $26 \mathrm{~S}$ ribosomal.

Canistrocarpus cervicornis f. pseudohamatus (Cribb) M.J. Wynne.

Distribución: Quintana Roo: litoral, insular (Mendoza-González et al., 2016).

Canistrocarpus crispatus (J.V. Lamouroux) De Paula \& De Clerck.

Distribución: Quintana Roo: litoral, insular (Mateo-Cid et al., 2019).

\section{Dictyopteris delicatula J.V. Lamouroux.}

Distribución: Tamaulipas: litoral (Ortega et al., 2001; Mendoza-González y Mateo-Cid, 2005); Veracruz: estuarino, litoral, insular (Sánchez-Rodríguez, 1980; Ortega et al., 2001; Mendoza-González y Mateo-Cid, 2005; Landa-Cansigno et al., 2019; como D. delicatula, J. C. Sánchez-Hernández s.n. (UAMIZ 1141)); Tabasco: litoral (Mendoza-González et al., 2017); Campeche: estuarino, litoral, insular (Díaz-Martín y Espinoza-Ávalos, 2000; Mendoza-González y Mateo-Cid, 2005; Mateo-Cid et al., 2013a, b; Mendoza-González et al., 2016); Yucatán: litoral, insular (Díaz-Martín y Espinoza-Ávalos, 2000; Ortega et al., 2001; Mendoza-González y Mateo-Cid, 2005; Mendoza-González et al., 2016); Quintana Roo: litoral, insular (Mateo-Cid y Mendoza-González, 1991; Dreckmann et al., 1996; Díaz-Martín y Espinoza-Ávalos, 2000; Ortega et al., 2001; Mendoza-González y Mateo-Cid, 2005; MendozaGonzález et al., 2007; Valadez-Cruz et al., 2014; MendozaGonzález et al., 2016; Mateo-Cid et al., 2019).

\section{Dictyopteris jamaicensis W.R. Taylor.}

Distribución: Veracruz: litoral (De la Cruz-Francisco et al., 2017); Campeche: estuarino, litoral, insular (Callejas Jiménez et al., 2005; Mateo-Cid et al., 2013a; M. Callejas s.n. (UAMIZ 928)); Yucatán: litoral (Díaz-Martín y Espinoza-Ávalos, 2000; Ortega et al., 2001; Mendoza-González y Mateo- 
Cid, 2005); Quintana Roo: litoral, insular (Díaz-Martín y Espinoza-Ávalos, 2000; Ortega et al., 2001; Mendoza-González y Mateo-Cid, 2005; Mendoza-González et al., 2016; Mateo-Cid et al., 2019).

Dictyopteris jolyana E.C. Oliveira \& R.P. Furtado.

Distribución: Quintana Roo: litoral, insular (Mendoza-González y Mateo-Cid, 2005; Mendoza-González et al., 2007; Mendoza-González et al., 2016).

Dictyopteris justii J.V. Lamouroux.

Distribución: Tamaulipas: litoral (Ortega et al., 2001; Mendoza-González y Mateo-Cid, 2005); Tabasco: litoral (Sentíes y Dreckmann, 2013); Campeche: litoral, insular (MendozaGonzález y Mateo-Cid, 2005; Mateo-Cid et al., 2013a; Mendoza-González et al., 2016); Yucatán: litoral (Díaz-Martín y Espinoza-Ávalos, 2000; Ortega et al., 2001; MendozaGonzález y Mateo-Cid, 2005); Quintana Roo: litoral, insular (Mateo-Cid y Mendoza-González, 1991; Dreckmann et al., 1996; Díaz-Martín y Espinoza-Ávalos, 2000; Ortega et al., 2001; Mendoza-González y Mateo-Cid, 2005; MendozaGonzález et al., 2007; Valadez-Cruz et al., 2014; MendozaGonzález et al., 2016; Mateo-Cid et al., 2019; A. Sentíes s.n. (UAMIZ 1128)).

Dictyopteris plagiogramma (Montagne) Vickers.

Distribución: Yucatán: litoral, insular (Díaz-Martín y Espinoza-Ávalos, 2000; Ortega et al., 2001; Mendoza-González y Mateo-Cid, 2005); Quintana Roo: litoral, insular (Díaz-Martín y Espinoza-Ávalos, 2000; Ortega et al., 2001; MendozaGonzález y Mateo-Cid, 2005; Mendoza-González et al., 2007).

Dictyopteris polypodioides (A.P. De Candolle) J.V. Lamouroux.

Distribución: Campeche: litoral, insular (Mateo-Cid et al., 2013a; Mendoza-González et al., 2016); Yucatán: litoral (Díaz-Martín y Espinoza-Ávalos, 2000; Ortega et al., 2001);
Quintana Roo: litoral, insular (Díaz-Martín y Espinoza-Ávalos, 2000; Ortega et al., 2001; Mendoza-González y MateoCid, 2005; Mendoza-González et al., 2016).

Dictyota bartayresiana J.V. Lamouroux.

= Dictyota bartayresii J.V. Lamouroux.

Distribución: Veracruz: estuarino, litoral, insular (MateoCid et al., 1996; Ortega et al., 2001; González Gándara et al., 2007; Godínez-Ortega et al., 2009; Robinson et al., 2012; Landa-Cansigno et al., 2019; como D. bartayresii, Sánchez Rodríguez, 1980; como D. bartayresii, L. GonzálezMaldonado s.n. (UAMIZ 371); Campeche: estuarino, litoral, insular (Díaz-Martín y Espinoza-Ávalos, 2000; Ortega et al., 2001; Mateo-Cid et al., 2013b; Mendoza-González et al., 2016; como D. batayresii, Ortega, 1995); Yucatán: estuarino, litoral, insular (Díaz-Martín y Espinoza-Ávalos, 2000; Ortega et al., 2001); Quintana Roo: litoral, insular (MateoCid y Mendoza-González, 1991; Dreckmann et al., 1996; Díaz-Martín y Espinoza-Ávalos, 2000; Ortega et al., 2001; Valadez-Cruz et al., 2014; Mendoza-González et al., 2016; Mateo-Cid et al., 2019; como D. bartayresii, K. M. Dreckmann y A. Sentíes s.n (UAMIZ 524).

Dictyota canariensis (Grunow) Tronholm.

Distribución: Veracruz: litoral (Lozano-Orozco et al., 2014; J. Lozano-Orozco s.n. (UAMIZ 1226)).

Dictyota caribaea Hörnig \& Schnetter.

Distribución: Veracruz: litoral (Luna Ortega y de la CruzFrancisco, 2017); Quintana Roo: litoral, insular (Cetz-Navarro et al., 2008; Valadez-Cruz et al., 2014; Mendoza-González et al., 2016; Luna Ortega y de la Cruz-Francisco, 2017; Mateo-Cid et al., 2019).

Dictyota chalchicueyecanensis Lozano-Orozco \& Sentíes.

Distribución: Veracruz: litoral (Lozano-Orozco et al., 2016; J. Lozano-Orozco s.n. (UAMIZ 1283, UAMIZ 1284, UAMIZ 1285). 
Dictyota ciliolata Sonder ex Kützing.

Distribución: Tamaulipas: litoral (Ortega et al., 2001); Veracruz: litoral, insular (Sánchez-Rodríguez, 1980; Ortega et al., 2001; Godínez-Ortega et al., 2009; De la CruzFrancisco et al., 2017; Landa-Cansigno et al., 2019; K. M. Dreckmann y M. L. Núñez-Reséndiz s.n. (UAMIZ 1227), J. Lozano-Orozco s.n. (UAMIZ 1286, UAMIZ 1293, UAMIZ 1294, UAMIZ 1295, UAMIZ 1356, UAMIZ 1357); Campeche: estuarino, litoral, insular (Ortega, 1995; Díaz-Martín y Espinoza-Ávalos, 2000; Ortega et al., 2001; Mateo-Cid et al., 2013b; Mendoza-González et al., 2016); Yucatán: litoral (Díaz-Martín y Espinoza-Ávalos, 2000; Ortega et al., 2001; Mendoza-González et al., 2016); Quintana Roo: litoral, insular (Mateo-Cid y Mendoza-González, 1991; Dreckmann et al., 1996; Díaz-Martín y Espinoza-Ávalos, 2000; Ortega et al., 2001; Valadez-Cruz et al., 2014; Mateo-Cid et al., 2019).

Dictyota crenulata J. Agardh.

= Dictyota ciliolata var. bermudensis Taylor.

Distribución: Veracruz: litoral (Luna Ortega y de la CruzFrancisco, 2017; como D. ciliolata var. bermudensis, Ortega et al., 2001); Tabasco: litoral (Mendoza-González et al., 2017); Quintana Roo: litoral, insular (Díaz-Martín y Espinoza-Ávalos, 2000; Mateo-Cid et al., 2019).

Dictyota dichotoma (Hudson) J.V. Lamouroux.

= Dictyota volubilis Kützing.

Distribución: Veracruz: estuarino, litoral (Sánchez-Rodríguez, 1980); Campeche: estuarino, litoral, insular (MateoCid et al., 2013a, b; Mendoza-González et al., 2016); Yucatán: litoral (Sánchez-Molina et al., 2007; como D. volubilis, Díaz-Martín y Espinoza-Ávalos, 2000); Quintana Roo: estuarino, litoral, insular (Mateo-Cid y Mendoza-González, 1991; Collado-Vides et al., 1995; Dreckmann et al., 1996; Valadez-Cruz et al., 2014; Mendoza-González et al., 2016; Mateo-Cid et al., 2019; como D. volubilis, Díaz-Martín y Es-
pinoza-Ávalos, 2000; Mendoza-González et al., 2007; como D. dichotoma, A. Sentíes y L. Morquecho s.n. (UAMIZ 536), K. M. Dreckmann s.n. (UAMIZ 841), R. Torres s.n. (UAMIZ 1143)).

Dictyota dichotoma var. intricata (C.Agardh) Greville.

Distribución: Quintana Roo: litoral (Valadez-Cruz et al., 2014; Mateo-Cid et al., 2019).

Dictyota fasciola (Roth) J.V. Lamouroux.

Distribución: Veracruz: litoral (Luna Ortega y de la CruzFrancisco, 2017).

\section{Dictyota friabilis Setchell.}

Distribución: Quintana Roo: litoral (Mateo-Cid et al., 2019).

Dictyota guajirae Hörnig, Schnetter \& J.M. Over.

Distribución: Quintana Roo: litoral (Mateo-Cid et al., 2019).

Dictyota guineënsis (Kützing) P. Crouan \& H. Crouan.

इDilophus guineensis (Kützing) J. Agardh.

Distribución: Veracruz: litoral, insular (Ortega et al., 2001; como D. guineensis, Sánchez-Rodríguez, 1980); Campeche: litoral (Díaz-Martín y Espinoza-Ávalos, 2000); Yucatán: litoral, insular (Díaz-Martín y Espinoza-Ávalos, 2000; Ortega et al., 2001); Quintana Roo: litoral, insular (Dreckmann et al., 1996; Díaz-Martín y Espinoza-Ávalos, 2000; Ortega et al., 2001; Valadez-Cruz et al., 2014; Mendoza-González et al., 2016; Mateo-Cid et al., 2019; como D. guineensis, MateoCid y Mendoza-González, 1991; J. Lozano-Orozco s.n. (UAMIZ 1355, UAMIZ 1359, UAMIZ 1364)).

\section{Dictyota hamifera Setchell.}

Distribución: Quintana Roo: litoral, insular (Cetz-Navarro et al., 2008; Mendoza-González et al., 2016). 
Dictyota humifusa Hörnig, Schnetter \& Coppejans.

Distribución: Veracruz: litoral (Godínez-Ortega et al., 2019).

Dictyota implexa (Desfontaines) J.V. Lamouroux.

= Dictyota divaricata J.V. Lamouroux.

= Dictyota linearis (C. Agardh) Greville.

Distribución: Veracruz: litoral, insular (como D. divaricata y D. linearis, Sánchez-Rodríguez, 1980; Ortega et al., 2001); Tabasco: litoral (Sentíes y Dreckmann, 2013); Campeche: estuarino, insular (como $D$. divaricata y $D$. linearis, Ortega et al., 2001; como D. linearis, M. Callejas s.n. (UAMIZ 930)); Yucatán: litoral, insular (como $D$. divaricata y $D$. linearis, Ortega et al., 2001); Quintana Roo: litoral, insular (MateoCid et al., 2019; como D. linearis y D. divaricata, Mateo-Cid y Mendoza-González, 1991; Ortega et al., 2001, como $D$. linearis, G. Castillo s.n. (UAMIZ 392), como D. divaricata, G. Castillo s.n. (UAMIZ 468)).

Dictyota jamaicensis W.R. Taylor.

Distribución: Veracruz: litoral, insular (Ortega et al., 2001; Anónimo s.n. (UAMIZ 1287); Campeche: litoral, insular (Díaz-Martín y Espinoza-Ávalos, 2000; Ortega et al., 2001; Mendoza-González et al., 2013; Mendoza-González et al., 2016); Yucatán: litoral, insular (Ortega et al., 2001; Mendoza-González et al., 2016); Quintana Roo: litoral, insular (Mateo-Cid y Mendoza-González, 1991; Dreckmann et al., 1996; Díaz-Martín y Espinoza-Ávalos, 2000; Ortega et al., 2001; Valadez-Cruz et al., 2014; Mendoza-González et al., 2016; J. Lozano-Orozco s.n. (UAMIZ 1361, UAMIZ 1362)).

Dictyota mayae Lozano-Orozco \& Sentíes.

Distribución: Quintana Roo: litoral (Lozano-Orozco et al., 2015; J. Lozano-Orozco s.n. (UAMIZ 1280)).

Dictyota menstrualis (Hoyt) Schnetter, Hörnig \& Weber-Peukert.

Distribución: Tamaulipas: estuarino, litoral (Ortega et al., 2001); Veracruz: estuarino, litoral, insular (Ortega et al.,
2001; González Gándara et al., 2007; Godínez-Ortega et al., 2009; Ramírez Rodríguez y Blanco Pérez, 2011; Robinson et al., 2012); Campeche: estuarino, litoral, insular (Díaz-Martín y Espinoza-Ávalos, 2000; Ortega et al., 2001; Mateo-Cid et al., 2013a, b; Mendoza-González et al., 2013; MendozaGonzález et al., 2016); Yucatán: estuarino, litoral, insular (Díaz-Martín y Espinoza-Ávalos, 2000; Ortega et al., 2001); Quintana Roo: estuarino, litoral, insular (Díaz-Martín y Espinoza-Ávalos, 2000; Ortega et al., 2001; Quan-Young et al., 2006; Mendoza-González et al., 2007; Ortegón-Aznar et al., 2009; Valadez-Cruz et al., 2014; Mendoza-González et al., 2016; Mateo-Cid et al., 2019).

Dictyota mertensii (C. Martius) Kützing.

= Dictyota dentata J.V. Lamouroux.

Distribución: Veracruz: litoral, insular (Ortega et al., 2001); Campeche: litoral (Díaz-Martín y Espinoza-Ávalos, 2000); Yucatán: litoral, insular (Díaz-Martín y Espinoza-Ávalos, 2000; Ortega et al., 2001); Quintana Roo: litoral, insular (Mateo-Cid y Mendoza-González, 1991; Dreckmann et al., 1996; Díaz-Martín y Espinoza-Ávalos, 2000; Ortega et al., 2001; Quan-Young et al., 2006; Mendoza-González et al., 2007; Valadez-Cruz et al., 2014; Mendoza-González et al., 2016; Mateo-Cid et al., 2019; como D. dentata, K. M. Dreckmann s.n. (UAMIZ 564), K. M. Dreckmann y A. Sentíes s.n. (UAMIZ 593)).

\section{Dictyota pedrochei Lozano-Orozco \& Sentíes.}

Distribución: Quintana Roo: litoral (Lozano-Orozco et al., 2015; J. Lozano-Orozco s.n. (UAMIZ 1281)).

Dictyota pinnatifida Kützing.

= Dilophus alternans J. Agardh.

= Dictyota alternans (J. Agardh) Hörnig, Schnetter \& Prud'homme.

Distribución: Veracruz: litoral (Luna Ortega y de la CruzFrancisco, 2017); Campeche: estuarino, litoral (Mateo-Cid et al., 2013b; Mendoza-González et al., 2016); Yucatán: in- 
sular (Ortega et al., 2001; Mendoza-González et al., 2016); Quintana Roo: litoral, insular (Díaz-Martín y EspinozaÁvalos, 2000; Ortega et al., 2001; Quan-Young et al., 2006; Valadez-Cruz et al., 2014; Mendoza-González et al., 2016; Mateo-Cid et al., 2019; J. Lozano-Orozco s.n. (UAMIZ 1360); como D. alternans, Mateo-Cid y Mendoza-González, 1991; Dreckmann et al., 1996; M. Gallegos s.n. (UAMIZ 1167).

\section{Dictyota pulchella Hörnig \& Schnetter.}

Distribución: Veracruz: litoral, insular (González Gándara et al., 2007; Robinson et al., 2012; Luna Ortega y de la Cruz-Francisco, 2017); Campeche: estuarino, litoral, insular (Díaz-Martín y Espinoza-Ávalos, 2000; Callejas Jiménez et al., 2005; Mateo-Cid et al., 2013a, b; Mendoza-González et al., 2013, 2016); Yucatán: litoral (Díaz-Martín y EspinozaÁvalos, 2000; Mendoza-González et al., 2016); Quintana Roo: litoral, insular (Díaz-Martín y Espinoza-Ávalos, 2000; Quan-Young et al., 2006; Mendoza-González et al., 2007; Cetz-Navarro et al., 2008; Valadez-Cruz et al., 2014; Mendoza-González et al., 2016; Mateo-Cid et al., 2019; F. Aguilar s.n. (UAMIZ 1093)).

Dictyota spiralis Montagne.

Distribución: Veracruz: litoral (Luna Ortega y de la CruzFrancisco, 2017).

Lobophora declerckii N.E. Schultz, C.W. Schneider \& L. Le Gall.

Distribución: Veracruz: insular (Godínez-Ortega et al., 2018).

Lobophora variegata (J.V. Lamouroux) Womersley ex E.C. Oliveira.

Distribución: Tamaulipas: litoral (Ortega et al., 2001); Veracruz: litoral, insular (Ortega et al., 2001; Díaz-Martínez et al., 2016); Campeche: litoral, insular (Díaz-Martín y Espinoza-Ávalos, 2000; Ortega et al., 2001; Mateo-Cid et al., 2013a; Mendoza-González et al., 2016); Yucatán: litoral, insular (Díaz-Martín y Espinoza-Ávalos, 2000; Ortega et al., 2001; Mendoza-González et al., 2016); Quintana Roo: estuarino, litoral, insular (Mateo-Cid y Mendoza-González, 1991; Dreckmann et al., 1996; Díaz-Martín y EspinozaÁvalos, 2000; Ortega et al., 2001; Mendoza-González et al., 2007; Valadez-Cruz et al., 2014; Mendoza-González et al., 2016; Godínez-Ortega et al., 2018; Mateo-Cid et al., 2019).

Padina antillarum (Kützing) Piccone.

Distribución: Tamaulipas: litoral (Díaz-Martínez et al., 2016); Campeche: estuarino, litoral (Mendoza-González et al., 2013; Díaz-Martínez et al., 2016).

Padina boergesenii Allender \& Kraft.

Distribución: Veracruz: estuarino, litoral, insular (MateoCid et al., 1996; Godínez-Ortega et al., 2009; Ávila-Ortiz et al., 2011; Díaz-Martínez et al., 2016; Landa-Cansigno et al., 2019); Campeche: estuarino, litoral, insular (Ortega, 1995; Díaz-Martín y Espinoza-Ávalos, 2000; Ortega et al., 2001; Ávila-Ortiz et al., 2011; Mateo-Cid et al., 2013b; MendozaGonzález et al., 2013; Díaz-Martínez et al., 2016; MendozaGonzález et al., 2016); Yucatán: litoral, insular (Díaz-Martín y Espinoza-Ávalos, 2000; Ortega et al., 2001; Ávila-Ortiz et al., 2011; Mendoza-González et al., 2016); Quintana Roo: litoral, insular (Mateo-Cid y Mendoza-González, 1991; DíazMartín y Espinoza-Ávalos, 2000; Ortega et al., 2001; ÁvilaOrtiz et al., 2011; Valadez-Cruz et al., 2014; Mendoza-González et al., 2016; Mateo-Cid et al., 2019).

Padina gymnospora (Kützing) Sonder.

= Padina vickersiae Hoyt.

Distribución: Tamaulipas: litoral (Ortega et al., 2001; F. F. Pedroche y A. Ávila s.n. (UAMIZ 1015)); Veracruz: estuarino, litoral, insular (Ortega et al., 2001; Godínez-Ortega et al., 2009; Ramírez Rodríguez y Blanco Pérez, 2011; González-Reséndiz et al., 2014; Díaz-Martínez et al., 2016; De la Cruz-Francisco et al., 2017; Landa-Cansigno et al., 2019; F. F. Pedroche y A. Ávila s.n. (UAMIZ 1014, UAMIZ 1016), A. Senties s.n. (UAMIZ 1148); como P. vickersiae, Sánchez Rodríguez, 1980; Campeche: estuarino, litoral (Ortega, 1995; Díaz-Martín y Espinoza-Ávalos, 2000; Ortega et al., 
2001; Callejas Jiménez et al., 2005; Mateo-Cid et al., 2013b; Mendoza-González et al., 2013; Díaz-Martínez et al., 2016; Mendoza-González et al., 2016; M. Callejas s.n. (UAMIZ 931, UAMIZ 939); Yucatán: estuarino, litoral, insular (DíazMartín y Espinoza-Ávalos, 2000; Ortega et al., 2001; Ortegón-Aznar et al., 2001; Díaz-Martínez et al., 2016; Mendoza-González et al., 2016); Quintana Roo: litoral, insular (Mateo-Cid y Mendoza-González, 1991; Dreckmann et al., 1996; Díaz-Martín y Espinoza-Ávalos, 2000; Ortega et al., 2001; Valadez-Cruz et al., 2014; Díaz-Martínez et al., 2016; Mendoza-González et al., 2016; Mateo-Cid et al., 2019; G. Castillo s.n. (UAMIZ 396)).

\section{Padina haitiensis Thivy.}

Distribución: Veracruz: insular (Ortega et al., 2001); Campeche: litoral (Mendoza-González et al., 2016); Quintana Roo: litoral, insular (Mateo-Cid y Mendoza-González, 1991; Ortega et al., 2001; Valadez-Cruz et al., 2014; MendozaGonzález et al., 2016; Mateo-Cid et al., 2019).

Padina pavonica (Linnaeus) Thivy.

Distribución: Tamaulipas: estuarino (Ortega et al., 2001); Veracruz: litoral, insular (Sánchez Rodríguez, 1980; Ortega et al., 2001; De la Cruz-Francisco et al., 2017; Robinson et al., 2012); Campeche: estuarino, litoral (Callejas Jiménez et al., 2005; Mateo-Cid et al., 2013b; Mendoza-González et al., 2013; Mendoza-González et al., 2016; M. Callejas s.n. (UAMIZ 933); Yucatán: litoral, insular (Díaz-Martín y Espinoza-Ávalos, 2000; Ortega et al., 2001; Mendoza-González et al., 2016); Quintana Roo: litoral, insular (Díaz-Martín y Espinoza-Ávalos, 2000; Ortega et al., 2001; Mendoza-González et al., 2016).

* Padina perindusiata Thivy.

Distribución: Veracruz: litoral (Ortega et al., 2001).

Padina profunda S.A. Earle.

Distribución: Campeche: insular (Mateo-Cid et al., 2013a).

\section{Padina sanctae-crucis Børgesen.}

= Dictyerpa jamaicensis Collins

= Padina jamaicensis (Collins) Papenfuss.

Distribución: Veracruz: litoral, insular (Sánchez Rodríguez, 1980; Ortega et al., 2001; González Gándara et al., 2007; Robinson et al., 2012; Díaz-Martínez et al., 2016; como D. jamaicensis, P. Rosales s.n. (UAMIZ 206, UAMIZ 208); Campeche: litoral (Díaz-Martín y Espinoza-Ávalos, 2000; Ortega et al., 2001; Mateo-Cid et al., 2013b; Díaz-Martínez et al., 2016; Mendoza-González et al., 2016); Yucatán: litoral, insular (Díaz-Martín y Espinoza-Ávalos, 2000; Ortega et al., 2001; Sánchez-Molina et al., 2007; Mendoza-González et al., 2016); Quintana Roo: litoral, insular (Díaz-Martín y Espinoza-Ávalos, 2000; Ortega et al., 2001; Mendoza-González et al., 2007; Valadez-Cruz et al., 2014; Díaz-Martínez et al., 2016; Mendoza-González et al., 2016; Mateo-Cid et al., 2019; M. Gallegos s.n. (UAMIZ 207, UAMIZ 216), A. Sentíes y L. Morquecho s.n. (UAMIZ 535), A. Senties y K. M. Dreckmann s.n. (UAMIZ 589), A. Sentíes s.n. (UAMIZ 651); como P. jamaicensis, Mateo-Cid y Mendoza-González, 1991; Dreckmann et al., 1996).

Spatoglossum schroederi (C. Agardh) Kützing.

Distribución: Tamaulipas: litoral (Ortega et al., 2001); Veracruz: estuarino, litoral, insular (Sánchez Rodríguez, 1980; Ortega et al., 2001; Godínez-Ortega et al., 2009; De la Cruz-Francisco et al., 2017; A. Sentíes s.n. (UAMIZ 1150)); Campeche: estuarino, insular (Mateo-Cid et al., 2013a, b; Mendoza-González et al., 2016); Yucatán: litoral, insular (Díaz-Martín y Espinoza-Ávalos, 2000; Ortega et al., 2001; Mendoza-González et al., 2016); Quintana Roo: litoral, insular (Mateo-Cid y Mendoza-González, 1991; Dreckmann et al., 1996; Díaz-Martín y Espinoza-Ávalos, 2000; Ortega et al., 2001; Valadez-Cruz et al., 2014; Mendoza-González et al., 2016; Mateo-Cid et al., 2019).

Stypopodium zonale (J.V. Lamouroux) Papenfuss.

Distribución: Tamaulipas: litoral (Ortega et al., 2001); Veracruz: estuarino, litoral (Sánchez Rodríguez, 1980; Ortega et 
al., 2001; Luna Ortega y de la Cruz-Francisco, 2017); Campeche: litoral, insular (Díaz-Martín y Espinoza-Ávalos, 2000; Mateo-Cid et al., 2013a, b); Yucatán: litoral, insular (DíazMartín y Espinoza-Ávalos, 2000; Ortega et al., 2001); Quintana Roo: litoral, insular (Mateo-Cid y Mendoza-González, 1991; Dreckmann et al., 1996; Díaz-Martín y EspinozaÁvalos, 2000; Ortega et al., 2001; Mendoza-González et al., 2007; Valadez-Cruz et al., 2014; Mendoza-González et al., 2016; Mateo-Cid et al., 2019; G. Castillo s.n. (UAMIZ 394), K. M. Dreckmann s.n. (UAMIZ 568, UAMIZ 855), A. Sentíes s.n. (UAMIZ 1127, UAMIZ 1129).

Zonaria tournefortii (J.V. Lamouroux) Montagne.

Distribución: Campeche: insular (Mateo-Cid et al., 2013a); Yucatán: litoral, insular (Díaz-Martín y Espinoza-Ávalos, 2000; Ortega et al., 2001; Mendoza-González et al., 2016); Quintana Roo: litoral, insular (Díaz-Martín y Espinoza-Ávalos, 2000; Ortega et al., 2001; Mendoza-González et al., 2016).

\section{Ectocarpales}

\section{Acinetosporaceae}

*Acinetospora crinita (Carmichael) Sauvageau.

Distribución: Veracruz: litoral (Sánchez Rodríguez, 1980; Ortega et al., 2001); Yucatán: litoral (Díaz-Martín y Espinoza-Ávalos, 2000; Ortega et al., 2001).

Feldmannia duchassaingiana (Grunow) Aisha \& M. Shameel.

इ Giffordia duchassaingiana (Grunow) W.R. Taylor.

Distribución: Veracruz: estuarino, litoral (como, G. duchassaingiana, Sánchez Rodríguez, 1980); Tabasco: litoral (Mendoza-González et al., 2017). Yucatán: litoral (Mendoza-González et al., 2016); Quintana Roo: litoral (Mendoza-González et al., 2016).

Comentarios taxonómicos: especie reportada en Veracruz como G. duchassaingiana, actualmente transferida al gé- nero Feldamannia Hamel, a partir de una revisión profunda de caracteres morfológicos poco variables en especies de Ectocarpales (Aisha y Shameel, 2011). Hasta el momento no se ha documentado la presencia de Giffordia Batters en la región.

*Feldmannia indica (Sonder) Womersley \& A. Bailey.

三 Giffordia indica (Sonder) Papenfuss \& Chihara.

Distribución: Tamaulipas: litoral (Ortega et al., 2001); Veracruz: estuarino, litoral (Ortega et al., 2001); Campeche: estuarino, litoral (Díaz-Martín y Espinoza-Ávalos, 2000; Ortega et al., 2001; como G. indica, Ortega, 1995); Yucatán: litoral (Díaz-Martín y Espinoza-Ávalos, 2000; Ortega et al., 2001); Quintana Roo: litoral (Díaz-Martín y Espinoza-Ávalos, 2000; Ortega et al., 2001).

Feldmannia irregularis (Kützing) Hamel.

Distribución: Veracruz: estuarino, litoral (Sánchez Rodríguez, 1980; Ortega et al., 2001; Nava-Olvera et al., 2017); Tabasco: litoral (Mendoza-González et al., 2017); Quintana Roo: litoral (Valadez-Cruz et al., 2014; Mateo-Cid et al., 2019).

Feldmannia mitchelliae (Harvey) H.-S. Kim.

इ Hincksia mitchelliae (Harvey) P.C. Silva.

三 Giffordia mitchelliae (Harvey) Hamel.

Distribución: Tamaulipas: litoral (como H. mitchelliae, Ortega et al., 2001); Veracruz: estuarino, litoral, insular (como G. mitchelliae, Sánchez Rodríguez, 1980; Ortega et al., 2001; como H. mitchelliae, Ramírez Rodríguez y Blanco Pérez, 2011); Tabasco: estuarino, litoral (Mendoza-González et al., 2017); Campeche: estuarino, litoral, insular (como $H$. mitchelliae, Ortega, 1995; Díaz-Martín y Espinoza-Ávalos, 2000; Ortega et al., 2001); Yucatán: litoral, insular (Mendoza-González et al., 2016; como H. mitchelliae, Díaz-Martín y Espinoza-Ávalos, 2000; Ortega et al., 2001); Quintana Roo: litoral, insular (Mendoza-González et al., 2016; Nava-Olvera et al., 2017; Mateo-Cid et al., 2019; como H. mitchelliae, 
Díaz-Martín y Espinoza-Ávalos, 2000; Ortega et al., 2001; Valadez-Cruz et al., 2014).

*Feldmannia padinae (Buffham) Hamel.

Distribución: Veracruz: estuarino, litoral (Sánchez Rodríguez, 1980; Ortega et al., 2001).

Herponema tortugense (W.R. Taylor) W.R. Taylor.

Distribución: Tabasco: estuarino (Mendoza-González et al., 2017); Campeche: insular (Mateo-Cid et al., 2013a); Quintana Roo: litoral, insular (Mendoza-González et al., 2007; Cetz-Navarro et al., 2008; Mendoza-González et al., 2016; Mateo-Cid et al., 2019).

Hincksia onslowensis (Amsler \& Kapraun) P.C. Silva.

Distribución: Quintana Roo: litoral (Cetz-Navarro et al., 2008; Mendoza-González et al., 2016).

*Hincksia sandriana (Zanardini) P.C.Silva.

Distribución: Quintana Roo: insular (Díaz-Martín y Espinoza-Ávalos, 2000).

Chordariaceae

Cladosiphon occidentalis Kylin.

Distribución: Veracruz: insular (Ortega et al., 2001; Robinson et al., 2012); Yucatán: litoral (Díaz-Martín y EspinozaÁvalos, 2000); Quintana Roo: litoral (Dreckmann et al., 1996; Díaz-Martín y Espinoza-Ávalos, 2000; Ortega et al., 2001; Valadez-Cruz et al., 2014).

Cladosiphon zosterae (J. Agardh) Kylin.

Distribución: Veracruz: litoral, insular (Mateo-Cid et al., 1996; Ortega et al., 2001; Godínez-Ortega et al., 2009; Nava-Olvera et al., 2017); Campeche: litoral (Mendoza-González et al., 2016); Yucatán: insular (Ortega et al., 2001); Quintana Roo: litoral, insular (Mateo-Cid y Mendoza-Gon- zález, 1991; Ortega et al., 2001; Nava-Olvera et al., 2017; Mateo-Cid et al., 2019).

\section{Ectocarpus confervoides Harvey.}

Distribución: Yucatán: litoral (Mendoza-González et al., 2016).

Ectocarpus rallsiae Vickers.

三 Hincksia rallsiae (Vickers) P.C. Silva.

三 Giffordia rallsiae (Vickers) W.R. Taylor.

Distribución: Tamaulipas: litoral (Robinson et al., 2012; Nava-Olvera et al., 2017; como H. rallsiae, Ortega et al., 2001); Veracruz: litoral, insular (como G. rallsiae, Sánchez Rodríguez, 1980; como H. rallsiae, Ortega et al., 2001; GodínezOrtega et al., 2009); Campeche: estuarino, litoral (como H. rallsiae, Mateo-Cid et al., 2013b; Mendoza-González et al., 2016); Yucatán: litoral (como H. rallsiae, Díaz-Martín y Espinoza-Ávalos, 2000; Mendoza-González et al., 2016); Quintana Roo: litoral, insular (como H. rallsiae, Díaz-Martín y Espinoza-Ávalos, 2000; Ortega et al., 2001).

Ectocarpus siliculosus (Dillwyn) Lyngbye.

$=$ Ectocarpus confervoides Le Jolis.

Distribución: Tamaulipas: estuarino (Ortega et al., 2001); Veracruz: estuarino, litoral (Ortega et al., 2001; Nava-Olvera et al., 2017; como E. confervoides, Sánchez Rodríguez, 1980); Tabasco: estuarino, litoral (Mendoza-González et al., 2017; Landa-Cansigno et al., 2019); Campeche: litoral, insular (Díaz-Martín y Espinoza-Ávalos, 2000; Ortega et al., 2001); Yucatán: litoral, insular (Díaz-Martín y Espinoza-Ávalos, 2000; Ortega et al., 2001); Quintana Roo: litoral, insular (Mateo-Cid y Mendoza-González, 1991; Díaz-Martín y Espinoza-Ávalos, 2000; Ortega et al., 2001; Mendoza-González et al., 2016).

Ectocarpus siliculosus var. dasycarpus (Kuckuck) Gallardo.

Distribución: Campeche: litoral (Mendoza-González et al., 2016); Yucatán: litoral (Mendoza-González et al., 2016). 
Ectocarpus variabilis Vickers.

Distribución: Veracruz: estuarino, litoral (Sánchez Rodríguez, 1980; Ortega et al., 2001); Yucatán: litoral, insular (Díaz-Martín y Espinoza-Ávalos, 2000; Ortega et al., 2001); Quintana Roo: litoral, insular (Mateo-Cid y Mendoza-González, 1991; Díaz-Martín y Espinoza-Ávalos, 2000; Ortega et al., 2001; Mendoza-González et al., 2016).

Hecatonema floridanum (W.R. Taylor) W.R. Taylor.

Distribución: Veracruz: litoral (Nava-Olvera et al., 2017); Tabasco: litoral (Mendoza-González et al., 2017); Quintana Roo: litoral (Nava-Olvera et al., 2017; Mateo-Cid et al., 2019).

*Hummia onusta (Kützing) J. Fiore.

= Ectocarpus subcorymbosus Farlow ex Holden.

Distribución: Tamaulipas: litoral (Ortega et al., 2001); Veracruz: estuarino, litoral (Ortega et al., 2001); Campeche: litoral, insular (Díaz-Martín y Espinoza-Ávalos, 2000; Ortega et al., 2001); Yucatán: litoral, insular (Díaz-Martín y Espinoza-Ávalos, 2000; Ortega et al., 2001); Quintana Roo: estuarino, litoral, insular (Díaz-Martín y Espinoza-Ávalos, 2000; Ortega et al., 2001; como E. subcorymbosus, Collado-Vides et al., 1995).

Kuetzingiella elachistaeformis (Heydrich) M. Balakrishnan \& Kinkar.

E Ectocarpus elachistaeformis Heydrich.

三 Feldmannia elachistaeformis (Heydrich) Pham-Hoàng Hô.

Distribución: Tamaulipas: litoral (como E. elachistaeformis, Ortega et al., 2001); Veracruz: estuarino, litoral (como E. elachistaeformis, Sánchez Rodríguez, 1980; Ortega et al., 2001); Tabasco: litoral (Mendoza-González et al., 2017); Campeche: estuarino, litoral (Mateo-Cid et al., 2013b; Mendoza-González et al., 2016; como F. elechistaeformis, Ortega, 1995; como E. elachistaeformis, Díaz-Martín y Espinoza-Ávalos, 2000; Ortega et al., 2001); Yucatán: litoral, insular (como E. elachistaeformis, Díaz-Martín y EspinozaÁvalos, 2000; Ortega et al., 2001); Quintana Roo: litoral, insular (Mendoza-González et al., 2007; Cetz-Navarro et al., 2008; Valadez-Cruz et al., 2014; Mendoza-González et al., 2016; Mateo-Cid et al., 2019; como E. elachistaeformis, Díaz-Martín y Espinoza-Ávalos, 2000; Ortega et al., 2001).

Levringia brasiliensis (Montagne) A.B. Joly.

Distribución: Yucatán: litoral (Mendoza-González et al., 2016).

Myrionema strangulans Greville.

Distribución: Veracruz: litoral (Nava-Olvera et al., 2017); Quintana Roo: litoral (Cetz-Navarro et al., 2008; MendozaGonzález et al., 2016; Mateo-Cid et al., 2019).

Nemacystus howei (W.R. Taylor) Kylin.

Distribución: Campeche: insular (Mateo-Cid et al., 2013a).

Streblonema invisibile Hoyt.

Distribución: Campeche: estuarino (Mateo-Cid et al., 2013b; Mendoza-González et al., 2016).

Scytosiphonaceae

Chnoospora minima (Hering) Papenfuss.

Distribución: Veracruz: estuarino, litoral, insular (Sánchez Rodríguez, 1980; Ortega et al., 2001; González-Reséndiz et al., 2014; De la Cruz-Francisco et al., 2017; Landa-Cansigno et al., 2019); Tabasco: litoral (Mendoza-González et al., 2017); Quintana Roo: litoral, insular (Mateo-Cid y Mendoza-González, 1991; Díaz-Martín y Espinoza-Ávalos, 2000; Ortega et al., 2001; Mendoza-González et al., 2016).

Colpomenia sinuosa (Mertens ex Roth) Derbès \& Solier.

Distribución: Tamaulipas: litoral (Ortega et al., 2001; Mendoza-González et al., 2017); Veracruz: estuarino, litoral, 
insular (Sánchez Rodríguez, 1980; Ortega et al., 2001; González Gándara et al., 2007; Aguilar-Rosas et al., 2008; Godínez-Ortega et al., 2009; Ramírez Rodríguez y Blanco Pérez, 2011; Robinson et al., 2012; González-Reséndiz et al., 2014; De la Cruz-Francisco et al., 2017; Landa-Cansigno et al., 2019); Tabasco: litoral (Mendoza-González et al., 2017); Campeche: litoral, insular (Díaz-Martín y Espinoza-Ávalos, 2000; Ortega et al., 2001; Aguilar-Rosas et al., 2008; Mateo-Cid et al., 2013a). Yucatán: litoral, insular (Díaz-Martín y Espinoza-Ávalos, 2000; Ortega et al., 2001; Aguilar-Rosas et al., 2008; Mendoza-González et al., 2016); Quintana Roo: litoral, insular (Mateo-Cid y Mendoza-González, 1991; Díaz-Martín y Espinoza-Ávalos, 2000; Ortega et al., 2001; Mendoza-González et al., 2007; Aguilar-Rosas et al., 2008; Mendoza-González et al., 2016; M. Gallegos s.n. (UAMIZ 1158)).

Hydroclathrus clathratus (C. Agardh) M. Howe.

Distribución: Veracruz: estuarino, litoral (Ortega et al., 2001; González Gándara et al., 2007; Anónimo s.n. (UAMIZ 1175)); Campeche: litoral (Díaz-Martín y Espinoza-Ávalos, 2000); Yucatán: litoral, insular (Díaz-Martín y Espinoza-Ávalos, 2000; Ortega et al., 2001); Quintana Roo: litoral, insular (Mateo-Cid y Mendoza-González, 1991; Díaz-Martín y Espinoza-Ávalos, 2000; Ortega et al., 2001; Mendoza-González et al., 2007; Mendoza-González et al., 2016).

*Petalonia fascia (O.F. Müller) Kuntze.

Distribución: Tamaulipas: litoral (Ortega et al., 2001).

*Planosiphon complanatus (Rosenvinge) McDevit \& G.W. Saunders.

इScytosiphon lomentaria var. complanatus Rosenvinge.

Distribución: Veracruz: insular (como S. lomentaria var. complanatus, Ortega et al., 2001).

Comentarios taxonómicos: especie recientemente transferida al género nuevo Planosiphon McDevit \& G.W. Saunders por McDevit y Saunders (2017); en su revisión de las especies de la familia Scytosiphonaceae presentes en Canadá, donde se evidenció la naturaleza parafilética del género Scytosiphon C. Agardh.

Rosenvingea endiviifolia (Martius) M.J. Wynne.

$=$ Rosenvingea intricata (J. Agardh) Børgesen.

Distribución: Tamaulipas: litoral (como R. intricata, Ortega et al., 2001); Veracruz: estuarino, litoral, insular (como R. intricata, Sánchez Rodríguez, 1980; Ortega et al., 2001; Godínez-Ortega et al., 2009; Robinson et al., 2012); Tabasco: estuarino, litoral (como $R$. intricata, Ortega et al., 2001; Sentíes y Dreckmann, 2013; Mendoza-González et al., 2017; Pérez-Jiménez et al., 2020; J. L. Arredondo s.n. (UAMIZ 300)); Yucatán: litoral (como R. intricata, Díaz-Martín y Espinoza-Ávalos, 2000); Quintana Roo: litoral, insular (como R. intricata, Mendoza-González et al., 2007; CetzNavarro et al., 2008, Mendoza-González et al., 2016).

Comentarios taxonómicos: especie puesta en sinonimia con $R$. endiviifolia en la revisión de Wynne y de Castro Nunes (2021), bajo esta nueva combinación, dado que Rosenvingea intricata es un nombre erróneamente aplicado a un mismo tipo taxonómico, cuya prioridad nomenclatural la tiene $R$. endiviifolia.

Rosenvingea floridana (W.R. Taylor) W.R. Taylor.

Distribución: Tabasco: litoral (Mendoza-González et al., 2017).

Rosenvingea orientalis (J. Agardh) Børgesen.

Distribución: Tabasco: litoral (Mendoza-González et al., 2017).

Rosenvingea sanctae-crucis $\mathrm{B} \emptyset$ rgesen.

Distribución: Veracruz: litoral (Nava-Olvera et al., 2017); Yucatán: litoral (Mendoza-González et al., 2016). 
Symphyocarpus strangulans Rosenvinge.

Distribución: Quintana Roo: litoral (Mateo-Cid et al., 2019).

Fucales

Sargassaceae

*Sargassum acinarium (Linnaeus) Setchell.

Distribución: Tamaulipas: litoral (Ortega et al., 2001); Veracruz: litoral Anónimo s.n. (UAMIZ 212).

Sargassum cymosum C. Agardh.

इ Sargassum hystrix var. buxifolium Chauvin.

= Sargassum bermudense Grunow.

= Sargassum buxifolium (Chauvin) M.J. Wynne.

= Sargassum filipendula C. Agardh.

= Sargassum filipendula var. laxum J. Agardh.

= Sargassum fluitans (Børgesen) Børgesen.

= Sargassum furcatum Kützing.

= Sargassum hystrix J. Agardh.

= Sargassum natans (Linnaeus) Gaillon.

= Sargassum polyceratium Montagne.

= Sargassum polyceratium var. ovatum (Collins) W.R. Taylor = Sargassum vulgare C. Agardh.

= Sargassum vulgare var. foliosissimum (J.V. Lamouroux) C. Agardh.

Distribución: Tamaulipas: litoral (como S. filipendula, S. fluitans, S. natans, S. vulgare, Ortega et al., 2001); Veracruz: estuarino, litoral, insular (como S. buxifolium, S. filipendula, S. fluitans, S. furcatum, S. natans, S. polyceratium, S. vulgare, Sánchez Rodríguez, 1980; como S. vulgare, S. filipendula, S. natans, Ortega et al., 2001; Godínez-Ortega et al., 2009; Ramírez Rodríguez y Blanco Pérez, 2011; como S. vulgare, Robinson et al., 2012; De la Cruz-Francisco et al., 2017; Landa-Cansigno et al., 2019; González-Nieto et al., 2020; F. Altamira s.n. (UAMIZ 123), A. Kobelkowsky s.n. (UAMIZ 209), P. Rosales (UAMIZ 214), R. Torres s.n. (UAMIZ
1117), A. Sentíes s.n. (UAMIZ 1119, UAMIZ 1255 , UAMIZ 1256), D. González-Nieto (UAMIZ 1032, UAMIZ 1033, UAMIZ 1034, UAMIZ 1038); Tabasco: litoral (como S. hystrix, S. natans, S. vulgare, Sentíes y Dreckmann, 2013); Campeche: estuarino, litoral, insular (como S. filipendula, $S$. fluitans, S. hystrix var. buxifolium, S. natans, Díaz-Martín y Espinoza-Ávalos, 2000; como S. buxifolium, S. filipendula, S. hystrix, S. natans, S. vulgare, Ortega et al., 2001; como S. filipendula, S. fluitans, S. furcatum, Mateo-Cid et al., 2013a, b; Mendoza-González et al., 2016); Yucatán: estuarino, litoral, insular (como S. buxifolium, S. filipendula, S. fluitans, S. furcatum, S. hystrix, S. natans, S. polyceratium, S. vulgare, Dreckmann et al., 1996; como S. filipendula, S. fluitans, S. furcatum, S. hystrix, S. hystrix var. buxifolium, S. natans, S. polyceratium var. ovatum, S. vulgare, Díaz-Martín y Espinoza-Ávalos, 2000; como S. filipendula, S. fluitans, S. natans, S. vulgare, Ortega et al., 2001; Ortegón-Aznar et al., 2001; Mendoza-González et al., 2016); Quintana Roo: litoral, insular (como S. buxifolium, S. filipendula, $S$. fluitans, S. furcatum, S. hystrix, S. natans, S. polyceratium, S. vulgare, Mateo-Cid y Mendoza-González, 1991; como S. filipendula, S. fluitans, S. furcatum, S. hystrix, S. hystrix var. buxifolium, S. natans, S. polyceratium, S. polyceratium var. ovatum, S. vulgare, Dreckmann et al., 1996; Díaz-Martín y Espinoza-Ávalos, 2000; como S. buxifolium, S. filipendula, S. fluitans, S. furcatum, S. hystrix, S. natans, S. polyceratium, S. vulgare, Ortega et al., 2001; Mateo-Cid et al., 2006; Quan-Young et al., 2006; Valadez-Cruz et al., 2014; como S. buxifolium, S. filipendula, S. fluitans, S. furcatum, S. natans, S. polyceratium var. ovatum, S. vulgare, Mendoza-González et al., 2016; como S. buxifolium, S. filipendula, S. fluitans,

S. hystrix, S. natans, S. polyceratium, S. polyceratium var. ovatum, S. vulgare, Mateo-Cid et al., 2019; González-Nieto et al., 2020; M. Gallegos s.n. (UAMIZ 213, UAMIZ 215), G. Castillo s.n. (UAMIZ 393, UAMIZ 405, UAMIZ 448, UAMIZ 449, UAMIZ 453), I. Stout y K.M. Dreckmann s.n. (UAMIZ 574, UAMIZ 670), D. González-Nieto s.n. (UAMIZ 1052, UAMIZ 1053, UAMIZ 1054), K. M. Dreckmann s.n. (UAMIZ 837, UAMIZ 871, UAMIZ 872, UAMIZ 873, UAMIZ 874), A. Sentíes s.n. (UAMIZ 842), D. González-Nieto y L. E. Mateo-Cid s.n. (UAMIZ 1065, UAMIZ 1066, UAMIZ 1067, UAMIZ 1068, UAMIZ 1069, UAMIZ 1070, UAMIZ 1075). 
Comentarios taxonómicos: en su revisión de las especies de Sargassum C. Agardh con distribución en el Atlántico mexicano a partir de tres marcadores moleculares del mismo número de genomas algales, González-Nieto et al. (2020) encontraron que 10 de las 17 morfoespecies, incluyendo sus variedades y formas, registradas actualmente en el área son molecularmente idénticas entre sí, y los morfotipos de dichos taxones corresponden con el espectro de plasticidad fenotípica de S. cymosum, por tener prioridad nomenclatural. Sin embargo, González-Nieto et al. (2020) no revisaron tipos, por lo que la propuesta continúa como tal. En dicha reducción de diversidad se encuentran: S. bermudense, S. buxifolium, S. cymosum, $S$. filipendula, S. fluitans, S. furcatum, S. hystrix, S. natans, S. polyceratium y S. vulgare.

\section{Sargassum platycarpum Montagne.}

Distribución: Quintana Roo: litoral, insular (Mateo-Cid y Mendoza-González, 1991; Dreckmann et al., 1996; DíazMartín y Espinoza-Ávalos, 2000; Ortega et al., 2001; Valadez-Cruz et al., 2014; Mendoza-González et al., 2016; Mateo-Cid et al., 2019; González-Nieto et al., 2020; D. González-Nieto s.n. (UAMIZ 211, UAMIZ 390, UAMIZ 566, UAMIZ 1050).

\section{Sargassum ramifolium Kützing.}

Distribución: Yucatán: litoral, insular (Díaz-Martín y Espinoza-Ávalos, 2000; Ortega et al., 2001); Quintana Roo: litoral, insular (Díaz-Martín y Espinoza-Ávalos, 2000, Ortega et al., 2001; Mendoza-González et al., 2007; Mendoza-González et al., 2016; González-Nieto et al., 2020; D. González-Nieto s.n. (UAMIZ 1056, UAMIZ 1062).

\section{Sargassum rigidulum Kützing.}

Distribución: Tamaulipas: litoral (Ortega et al., 2001); Veracruz: estuarino, litoral (Sánchez Rodríguez, 1980; Ortega et al., 2001; González-Nieto et al., 2020; D. González-Nieto s.n. (UAMIZ 1026, UAMIZ 1039)); Tabasco: litoral (Sentíes y Dreckmann, 2013); Campeche: estuarino, litoral, insular
(Mateo-Cid et al., 2013a; M. Callejas s.n. (UAMIZ 929)); Yucatán: insular (Ortega et al., 2001); Quintana Roo: estuarino, litoral, insular (Mateo-Cid y Mendoza-González, 1991; Dreckmann et al., 1996; G. Castillo s.n. (UAMIZ 454); Ortega et al., 2001; Mateo-Cid et al., 2006; Quan-Young et al., 2006; Mateo-Cid et al., 2019; González-Nieto et al., 2020; D. González-Nieto y L.E. Mateo-Cid s.n. (UAMIZ 1057, UAMIZ 1071, UAMIZ 1072)).

Sargassum xochitliae González-Nieto, M.C. Oliveira \& Mateo-Cid.

Distribución: Quintana Roo: insular (González-Nieto et al., 2020).

Turbinaria tricostata E.S. Barton.

Distribución: Campeche: litoral, insular (Díaz-Martín y Espinoza-Ávalos, 2000; Ortega et al., 2001); Yucatán: litoral, insular (Díaz-Martín y Espinoza-Ávalos, 2000; Ortega et al., 2001); Quintana Roo: litoral, insular (Mateo-Cid y Mendoza-González, 1991; Dreckmann et al., 1996; Díaz-Martín y Espinoza-Ávalos, 2000; Ortega et al., 2001; Valadez-Cruz et al., 2014; Mendoza-González et al., 2016; Mateo-Cid et al., 2019; González-Nieto et al., 2020; G. Castillo s.n. (UAMIZ 404, UAMIZ 450), D. González-Nieto y L. E. Mateo-Cid s.n. (UAMIZ 1079, UAMIZ 1080, UAMIZ 1081, UAMIZ 1082, UAMIZ 1083).

\section{Turbinaria turbinata (Linnaeus) Kuntze.}

Distribución: Campeche: litoral, insular (Díaz-Martín y Espinoza-Ávalos, 2000; Ortega et al., 2001); Yucatán: litoral, insular (Díaz-Martín y Espinoza-Ávalos, 2000; Ortega et al., 2001); Quintana Roo: litoral, insular (Mateo-Cid y Mendoza-González, 1991; Dreckmann et al., 1996; Díaz-Martín y Espinoza-Ávalos, 2000; Ortega et al., 2001; Valadez-Cruz et al., 2014; Mendoza-González et al., 2016; Mateo-Cid et al., 2019; González-Nieto et al., 2020; G. Castillo s.n. (UAMIZ 403, UAMIZ 452), I. Stout y K. M. Dreckmann s.n. (UAMIZ 573), K. M. Dreckmann s.n. (UAMIZ 869), D. González- Nieto s.n. (UAMIZ 1078). 
Onslowiales

\section{Onslowiaceae}

Onslowia bahamensis E.C. Henry.

Distribución: Campeche: litoral (Mendoza-González et al., 2013); Quintana Roo: litoral (Cetz-Navarro et al., 2008; Mendoza-González et al., 2016).

Onslowia endophytica Searles.

Distribución: Campeche: litoral (Mendoza-González et al., 2016); Quintana Roo: litoral (Valadez-Cruz et al., 2014; Mendoza-González et al., 2016; Mateo-Cid et al., 2019).

Verosphacela ebrachia E.C. Henry.

Distribución: Quintana Roo: litoral, insular (Mateo-Cid et al., 2002; Mendoza-González et al., 2007; Cetz-Navarro et al., 2008; Mendoza-González et al., 2016).

Ralfsiales

Neoralfsiaceae

Neoralfsia expansa (J. Agardh) P.E. Lim \& H. Kawai ex Cormaci \& G. Furnari.

$\equiv$ Ralfsia expansa (J. Agardh) J. Agardh.

Distribución: Veracruz: estuarino, litoral (León-Álvarez et al., 2014a; como R. expansa, Sánchez Rodríguez, 1980); Campeche: litoral (Mendoza-González et al., 2016); Yucatán: litoral, insular (Mendoza-González et al., 2016; como R. expansa, Díaz-Martín y Espinoza-Ávalos, 2000; Ortega et al., 2001); Quintana Roo: litoral, insular (como R. expansa, Dreckmann et al., 1996; Díaz-Martín y Espinoza-Ávalos, 2000; Ortega et al., 2001).

Comentarios taxonómicos: especie transferida como nueva combinación al nuevo género Neoralfsia P.-E. Lim \& H. Kawai (León-Álvarez et al., 2014a), descrito para resolver la parafilia al interior de Ralfsia Berkeley, en el cual este se encontraba clasificada. Actualmente no existe evidencia molecular que sustente la presencia de Ralfsia en el Atlántico mexicano.

Scytothamnales

\section{Asteronemataceae}

Asteronema breviarticulatum (J. Agardh) Ouriques \& Bouzon.

इ Hincksia breviarticulata (J. Agardh) P.C. Silva.

इEctocarpus breviarticulatus J. Agardh.

Distribución: Veracruz: estuarino, litoral (González-Reséndiz et al., 2014; Landa-Cansigno et al., 2019; como E. breviarticulatus, Sánchez Rodríguez, 1980; como H. breviarticulata, Ortega et al., 2001); Tabasco: litoral (Mendoza-González et al., 2017); Yucatán: litoral, insular (como H. breviarticulata, Díaz-Martín y Espinoza-Ávalos, 2000; Ortega et al., 2001); Quintana Roo: litoral, insular (MendozaGonzález et al., 2016; como E. breviarticulatus, Mateo-Cid y Mendoza-González, 1991; como H. breviarticulata, DíazMartín y Espinoza-Ávalos, 2000; Ortega et al., 2001).

\section{Bachelotiaceae}

Bachelotia antillarum (Grunow) Gerloff.

Distribución: Veracruz: estuarino, litoral (Sánchez Rodríguez, 1980; Ortega et al., 2001); Tabasco: litoral (MendozaGonzález et al., 2017); Quintana Roo: litoral (Nava-Olvera et al., 2017).

\section{Sphacelariales}

Sphacelariaceae

Sphacelaria brachygonia Montagne.

Distribución: Yucatán: litoral, insular (Díaz-Martín y Espinoza-Ávalos, 2000; Ortega et al., 2001); Quintana 
Roo: litoral, insular (Mendoza-González y Mateo-Cid, 2000; Cetz-Navarro et al., 2008; Mendoza-González et al., 2016).

*Sphacelaria fusca (Hudson) S.F. Gray.

Distribución: Yucatán: litoral, insular (Díaz-Martín y Espinoza-Ávalos, 2000; Mendoza-González y Mateo-Cid, 2000; Ortega et al., 2001); Quintana Roo: litoral, insular (DíazMartín y Espinoza-Ávalos, 2000; Mendoza-González y Mateo-Cid, 2000; Ortega et al., 2001).

\section{Sphacelaria novae-hollandiae Sonder.}

Distribución: Veracruz: litoral, insular (Sánchez Rodríguez, 1980; Mendoza-González y Mateo-Cid, 2000; Ortega et al., 2001); Yucatán: litoral (Díaz-Martín y Espinoza-Ávalos, 2000; Mendoza-González et al., 2016); Quintana Roo: litoral, insular (Díaz-Martín y EspinozaÁvalos, 2000; Mendoza-González y Mateo-Cid, 2000; Ortega et al., 2001; Mendoza-González et al., 2016; MateoCid et al., 2019).

\section{Sphacelaria rigidula Kützing.}

= Sphacelaria furcigera Kützing.

Distribución: Tamaulipas: estuarino (Ortega et al., 2001); Veracruz: litoral, insular (Mendoza-González y Mateo-Cid, 2000; Ortega et al., 2001; Robinson et al., 2012; González-Reséndiz et al., 2014; como S. furcigera, Sánchez Rodríguez, 1980); Tabasco: litoral (Mendoza-González et al., 2017); Campeche: insular (Mateo-Cid et al., 2013a); Yucatán: litoral, insular (Díaz-Martín y Espinoza-Ávalos, 2000; Mendoza-González y Mateo-Cid, 2000; Ortega et al., 2001; Mendoza-González et al., 2016); Quintana Roo: litoral, insular (Mateo-Cid y Mendoza-González, 1991; Díaz-Martín y Espinoza-Ávalos, 2000; Mendoza-González y Mateo-Cid, 2000; Ortega et al., 2001; Mendoza-González et al., 2007; Mendoza-González et al., 2016; Nava-Olvera et al., 2017; Mateo-Cid et al., 2019).

\section{Sphacelaria tribuloides Meneghini.}

Distribución: Veracruz: estuarino, litoral, insular (Sánchez Rodríguez, 1980; Mateo-Cid et al., 1996; Mendoza-González y Mateo-Cid, 2000; Ortega et al., 2001; GonzálezReséndiz et al., 2014; Luna Ortega y de la Cruz-Francisco, 2017); Tabasco: litoral (Mendoza-González et al., 2017); Campeche: litoral, insular (Díaz-Martín y Espinoza-Ávalos, 2000; Ortega et al., 2001); Yucatán: litoral, insular (DíazMartín y Espinoza-Ávalos, 2000; Mendoza-González y Mateo-Cid, 2000; Ortega et al., 2001); Quintana Roo: litoral, insular (Mateo-Cid y Mendoza-González, 1991; Díaz-Martín y Espinoza-Ávalos, 2000; Mendoza-González y Mateo-Cid, 2000; Ortega et al., 2001; Mendoza-González et al., 2007; Valadez-Cruz et al., 2014; Mendoza-González et al., 2016; Mateo-Cid et al., 2019).

\section{Sporochnales}

\section{Sporochnaceae}

Nereia tropica (W.R. Taylor) W.R. Taylor.

Distribución: Campeche: insular (Mateo-Cid et al., 2013a).

Sporochnus pedunculatus (Hudson) C. Agardh.

Distribución: Tabasco: litoral (Sentíes y Dreckmann, 2013); Campeche: litoral, insular (Díaz-Martín y Espinoza-Ávalos, 2000; Mateo-Cid et al., 2013a; Mendoza-González et al., 2016); Yucatán: litoral, insular (Díaz-Martín y Espinoza-Ávalos, 2000; Ortega et al., 2001); Quintana Roo: litoral F. Aguilar s.n. (UAMIZ 1088), A. Kobelkoswky s.n. (UAMIZ 1089).

\section{Discusión}

De las 97 especies y 3 categorías infraespecíficas incluidas en este listado, la mayor riqueza especifica se encontró en el orden Dictyotales (43), seguido por Ectocarpales (33) y Fucales (8). Los órdenes menos diversos fueron Sphacelariales (5), Onslowiales (3), Scytothamniales y Sporochnales (con dos especies cada uno); Asterocladales y Ralfsiales (con 
una). No obstante, en órdenes con alta diversidad como Dictyotales, de las 43 especies registradas, 23 correspondieron al género Dictyota J.V. Lamouroux; en Fucales, de ocho conocidas, seis pertenecen a Sargassum C. Agardh. A pesar de su potencial económico debido a la presencia de alginatos en la pared celular, en México se conoce muy poco sobre la diversidad de este grupo, a diferencia de las algas rojas, también económicamente importantes, que han sido ampliamente estudiadas en la región desde trabajos florísticos hasta filogenéticos con descripciones de nuevas especies o filogeográficos (García-García et al., 2020).

En gran parte, esta marcada diferencia en la distribución de la diversidad puede explicarse, por un lado, porque Sargassum y Dictyota son de los géneros más conspicuos en el área tanto por sus tallas, que son mayores a los 10 cm (Vilchis et al., 2018), como por su abundancia y distribución en las costas del Golfo de México y Caribe mexicano (Dreckmann y Sentíes, 2013), y por otro lado porque han sido poco estudiados con un enfoque molecular. De los 45 trabajos revisados para la actualización del listado (Cuadro 1), solo siete incluyeron análisis de este tipo en algas pardas mexicanas, a pesar de que han producido cambios taxonómicos $y$, consecuentemente, en la diversidad de taxones registrados en el área. Los estudios en Dictyota (LozanoOrozco et al., 2014, 2015, 2016) revelaron que en un espectro morfológico poco variable hay una gran diversidad genética; como resultado, se han descrito nuevas especies mexicanas en este género, incrementando así la diversidad del grupo y la cantidad de registros para el Atlántico en general.

Estudios en Neoralfsia P.E. Lin \& H. Kawai (León-Álvarez et al., 2014a), Padina Adanson (Díaz-Martínez et al., 2016) y Lobophora J. Agardh (Godínez-Ortega et al., 2018) también aportaron cambios al estatus de algunas especies previamente registradas en el área, nuevas combinaciones e incorporación de registros nuevos. Contrariamente, en Sargassum (González-Nieto et al., 2020) se encontró que los taxones exhiben un espectro morfológico muy variable y marcado; al menos diez morfoespecies, con sus variedades, registradas continuamente en el área son genéticamente iguales y su plasticidad fenotípica se atribuyó a un solo taxón: S. cymosum. Este, al tener prioridad nomenclatural, repercute en una drástica reducción de diversidad es- pecífica en esta área. Sin embargo, con base en evidencias moleculares también se ha descrito una nueva especie de Sargassum (González-Nieto et al., 2020). Los restantes órdenes y géneros incluidos en el listado del presente trabajo carecen de análisis moleculares, por lo que es posible que al interior de Phaeophyceae aún exista diversidad por conocer y documentar.

Otro aspecto relevante son las diez especies que no han vuelto a ser registradas en listados florísticos de 2001 al presente o que fueron mencionadas una sola ocasión, al igual que en la revisión de algas rojas del Atlántico (GarcíaGarcía et al., 2020). Esto evidencia por un lado el cambio que tiene la flora en el tiempo, ya que no es estática, y por otro que los cambios taxonómicos y nomenclaturales en todos los niveles jerárquicos han incrementado el número de taxones en algunos grupos, o bien, su aplicación errónea en la ficoflora mexicana es razón por la cual algunas especies no han vuelto a citarse. En los listados revisados, la identificación taxonómica está basada en morfología, por lo que, hasta ahora, la flora conocida está integrada en su mayoría por morfoespecies que corresponden con descripciones de taxones cuyo intervalo de distribución se encuentran fuera de México, haciendo necesaria una revisión de esos casos para confirmar la identidad de esos registros o su ausencia en las costas mexicanas.

En cuanto a su distribución en el Atlántico mexicano, $62 \%$ de las especies han sido registradas tanto en el Golfo de México como en el Caribe mexicano, mientras que $23 \%$ son exclusivas del Golfo y $15 \%$ solo están en el Caribe. El alto porcentaje de taxones compartidos entre ambas regiones sugiere un elevado intercambio florístico, pero también, aquellos que no se comparten reflejan cierto aislamiento geográfico del Golfo de México y particularmente de la Sonda de Campeche. Como se ha discutido y evidenciado en estudios previos (Vilchis et al., 2018), su historia geológica ha tenido repercusiones en la corriente que pasa por el canal de Yucatán, la cual se desvía hacia el oeste bañando las costas del centro de Veracruz, sin tener influencia sobre el litoral de Campeche (Dreckmann y Sentíes, 2013; Vilchis et al., 2018). Como resultado, su comportamiento y composición florística ubican al litoral de Quintana Roo como fuente proveedora de especies al Golfo (Vilchis et al., 2018). En cuanto a distribución por estado, Quintana Roo 
posee la mayor diversidad de algas pardas en la región (75 especies), seguido por Veracruz con 57, Yucatán con 54, Campeche con 48 y la menor cantidad se encuentra en Tabasco y Tamaulipas con 25 y 23, respectivamente.

Las diferencias en la riqueza específica de cada estado indican que algunos presentan una mayor heterogeneidad ambiental para el establecimiento de comunidades algales, como es el caso de Quintana Roo, donde además el porcentaje de especies insulares (32\%) fue mayor que en otras entidades, en las que predominaron ambientes litorales (54\%). Tamaulipas tiene playas arenosas, mientras que Tabasco es principalmente estuarino; existen pocos sitios con sustrato rocoso donde las algas puedan establecerse, además, los constantes aportes de agua dulce que reciben las costas tabasqueñas modifican las condiciones de salinidad requeridas para el desarrollo de un gran número de algas pardas (Sentíes y Dreckmann, 2013).

\section{Conclusiones}

Aunque las feofitas son el grupo menos diverso dentro de las macroalgas marinas, también han sido menos atendidas. Por ello, es necesario continuar realizando estudios florísticos combinados con análisis moleculares que respalden un conocimiento más certero e integral de los recursos algales mexicanos y a su vez, posibiliten la incursión en investigaciones de manejo sustentable, utilización de recursos o propuestas de conservación de estos grupos, ya que, actualmente no son reconocidos o incorporados en ninguna ley de protección, y tampoco se sabe mucho sobre su potencial económico.

\section{Contribución de autores}

MLNR, KMD y AS concibieron y diseñaron el estudio. AMEGG y ECB realizaron la recopilación de datos, análisis de la información e integración de la base de datos y resultados. Todos los autores contribuyeron en la redacción, discusión, revisión y aprobación del manuscrito final.

\section{Financiamiento}

Este estudio fue apoyado por los proyectos: UAMICBS2019-2022: sesión 15.18-281118 y UAMI-CA-117, Programa para el Desarrollo Profesional Docente de la Secretaría de Educación Pública (PRODEP-SEP).

\section{Agradecimientos}

A los revisores anónimos por su importante contribución para mejorar el manuscrito en su versión actual.

\section{Literatura citada}

Abascal, A. J., J. Sheinbaum, J. Candela, J. Ochoa y A. Badan. 2003. Analysis of flow variability in the Yucatan Channel. Journal of Geophysical Research 108(C12): 3381. DOI: https://doi. org/10.1029/2003jc001922

Adl, S. M., A. G. B. Simpson, C. E. Lane, J. Lukeš, D. Bass, S. S. Bowser, M. W. Brown, F. Burki, M. Dunthorn, V. Hampl, A. Heiss, M. Hoppenrath, E. Lara, L. Le Gall, D. H. Lynn, H. McManus, E. A. D. Mitchell, S. E. Mozley-Stanridge, L. W. Parfrey, J. Pawlowski, S. Rueckert, L. Shadwick, C. L. Schoch, A. Smirnov y F. W. Spiegel. 2012. The Revised Classification of Eukaryotes. The Journal of Eukaryotic Microbiology 59(5): 429-493. DOI: https://doi.org/10.1111/j.15507408.2012.00644.x

Aguilar-Rosas, L. E., R. Aguilar-Rosas, F. F. Pedroche, C. Mendoza González y L. E. Mateo Cid. 2008. El género Colpomenia (Scytosiphonaceae, Phaeophycota) de las costas de México. In: Sentíes, A. y K. M. Dreckmann (eds.). Monografías Ficológicas, Vol. 3. Universidad Autónoma MetropolitanaIztapalapa y Universidad Autónoma de Baja California. Cd. Mx, México. Pp. 1-26. DOI: https://doi.org/10.3926/ oms.400

Aisha, K. y M. Shameel. 2011. Taxonomic study of the order Ectocarpales (Phaeophycota) from the coastal waters of Pakistan. International Journal on Algae 13(2): 128-148. DOI: https://doi.org/10.1615/interjalgae.v13.i2.40

Ávila-Ortiz, A. G., L. E. Mateo-Cid y A. C. Mendoza-González. 2011. Caracterización morfológica de Padina boergesenii (Dictyotaceae, Phaeophyceae) en la costa mexicana del Golfo de México y Mar Caribe. Polibotánica 31: 1-20.

Berov, D., E. Ballesteros, M. Sales y M. Verlaque. 2015. Reinstatement of species rank for Cystoseira bosphorica Sauvageau (Sargassaceae, Phaeophyceae). Cryptogamie, Algologie 36(1): 65-80. DOI: https://doi.org/10.7872/crya. v36.iss1.2015.65

Boisset, F., P. P. Ferrer-Gallego, G. Furnari, M. Cormaci y B. Dennetiere. 2016. Typification of the Mediterranean endemic deep-water macroalga Laminaria rodriguezii Bornet (Laminariaceae, Phaeophyceae). Cryptogamie, 
Algologie 37(2): 121-132. DOI: https://doi.org/10.7872/ crya/v37.iss2.2016.121

Boo, S. M., K. M. Lee, G. Y. Cho y W. Nelson. 2011. Colpomenia claytonii sp. nov. (Scytosiphonaceae, Phaeophyceae) based on morphology and mitochondrial cox3 sequences. Botanica Marina 54(2): 159-167. DOI: https://doi.org/10.1515/ bot.2011.017

Callejas Jiménez, M. E., A. Sentíes Granados y K. M. Dreckmann. 2005. Macroalgas bentónicas de Puerto Real, Faro de Santa Rosalía y Playa Preciosa, Campeche, México, con algunas consideraciones florísticas y ecológicas para el estado. Hidrobiológica 15(1): 89-96.

Cetz-Navarro, N. P., J. Espinoza-Ávalos, A. Sentíes-Granados y L. I. Quan-Young. 2008. Nuevos registros de macroalgas para el Atlántico mexicano y riqueza florística del Caribe mexicano. Hidrobiológica 18(1): 11-19.

Collado-Vides, L., J. González-González y E. Ezcurra. 1995. Patrones de distribución ficoflorística en el sistema lagunar de Nichupté, Quintana Roo, México. Acta Botanica Mexicana 31: 19-32. DOI: https://doi.org/10.21829/abm31.1995.734

De Clerck, O., F. Leliaert, H. Verbruggen, C. E. Lane, J. C. De Paula, D. I. Payo y E. Coppejans. 2006. A revised classification of the Dictyoteae (Dictyotales, Phaeophyceae) based on $r b c \mathrm{~L}$ and 265 ribosomal DNA sequence data analyses. Journal of Phycology 42(6): 1271-1288. DOI: https://doi.org/10.1111/ j.1529-8817.2006.00279.x

De la Cruz-Francisco, V., R. E. Orduña-Medrano, J. E. ParedesFlores, R. I. Vázquez-Estrada, M. González-González y L. Flores-Galicia. 2017. Una aproximación a la florística y faunística de la costa rocosa El Pulpo, Cazones, Veracruz, México. CICIMAR Oceánides 32(1): 39-58.

Díaz-Martín, M. A. y J. Espinoza-Ávalos. 2000. Distribution of brown seaweeds (Phaeophyta) in the Yucatán Peninsula, Mexico. Bulletin of Marine Science 66(2): 279-289.

Díaz-Martínez, S., G. C. Zuccarello, A. G. Salazar Chávez, F. F. Pedroche y A. G. Ávila-Ortiz. 2016. Species of Padina (Dictyotales, Phaeophyceae) in tropical Mexican waters based on molecular-assisted taxonomy. Phycologia 55(6): 673-687. DOI: https://doi.org/10.2216/16-15.1

Dreckmann, K. M. 1998. Clasificación y nomenclatura de las macroalgas marinas bentónicas del Atlántico Mexicano. Comisión Nacional para el Conocimiento y Uso de la Biodiversidad (CONABIO). México, D.F., México. 149 pp.
Dreckmann, K. M. y A. Sentíes. 2013. Las arribazones de algas marinas en el Caribe Mexicano: Evento biológico natural o basura en la playa. Biodiversitas 107: 7-11.

Dreckmann, K. M., I. Stout y A. Sentíes Granados. 1996. Lista actualizada de las algas marinas bentónicas de Puerto Morelos, Quintana Roo, Caribe Mexicano. Polibotánica 3: 1-17.

García-García, A. M. E., E. Cabrera-Becerril, M. L. Núñez-Reséndiz, K. M. Dreckmann y A. Sentíes. 2020. Actualización taxonómica de las algas rojas (Rhodophyta) marinas bentónicas del Atlántico mexicano. Acta Botanica Mexicana 127: e1677. DOI: https://doi.org/10.21829/abm127.2020.1677

Godínez-Ortega, J. L., P. Ramírez-García y K. Pedraza-Venegas. 2009. Cambios en la flora béntica de Arrecife Hornos (Veracruz, México). TIP Revista Especializada en Ciencias Químico-Biológicas 12(2): 59-65.

Godínez-Ortega, J. L., P. Ramírez-García, A. Granados-Barba y M. J. Wynne. 2019. New records of subtidal benthic marine algae from the state of Veracruz, southern Gulf of Mexico. Revista Mexicana de Biodiversidad 90: 1-17. DOI: https:// doi.org/10.22201/ib.20078706e.2019.90.2719

Godínez-Ortega, J. L., L. I. Cabrera, R. García-Sandoval, M. J. Wynne, H. F. Olivares-Rubio, P. Ramírez-García y A. Granados-Barba. 2018. Morphological and molecular characterization of Lobophora declerckii and L. variegata (Dictyotales, Ochrophyta) on the Atlantic coast of Mexico. Phytotaxa 382(1): 57-73. DOI: https://doi.org/10.11646/ phytotaxa.382.1.2

González Gándara, C., M. Cruz Arellano, C. Domínguez Barradas, A. Serrano Solís y A. de J. Basañez Muñoz. 2007. Macroalgas asociadas a cuatro hábitats del arrecife Tuxpan, Veracruz, México. Revista UDO Agrícola 7(1): 252-257. DOI: https:// doi.org/10.22201/ib.20078706e.2009.001.576

González-Nieto, D., M. C. Oliveira, M. L. Núñez-Resendiz, K. M. Dreckmann, L. E. Mateo-Cid y A. Sentíes. 2020. Molecular assessment of the genus Sargassum (Fucales, Phaeophyceae) from the Mexican coasts of the Gulf of Mexico and Caribbean, with the description of $S$. xochitlae sp. nov. Phytotaxa 461(4): 254-274. DOI: https://doi. org/10.11646/phytotaxa.461.4.3

González-Reséndiz, M. L., L. M. García-Sánchez, J. G. Rodríguez-Juárez, J. S. De Gyves-López y H. León Tejera. 2014. Caracterización de ambientes algales en Playa 
Muñecos, Veracruz, México. Investigación Universitaria Multidisciplinaria 13(13): 36-42.

Graham, L. E. y L. W. Wilcox. 2000. Algae. Prentice-Hall, Inc. Upper Saddle River, USA. 640 pp.

Guiry, M. D. y G. M. Guiry. 2021. AlgaeBase World-wide electronic publication. National University of Ireland. Galway, Ireland. https://www.algaebase.org (consultado agosto de 2021).

Landa-Cansigno, C., L. E. Mateo-Cid, A. C. Mendoza-González y J. A. Guerrero-Analco. 2019. Macroalgas marinas del litoral rocoso Neovolcánico de Veracruz, México. Acta Botanica Mexicana 126: e1525. DOI: https://doi.org/10.21829/ abm126.2019.1525

León-Álvarez, D., M. L. Núñez-Resendiz y M. J. Wynne. 2014a. Morphological and molecular studies on topotype material of Neoralfsia expansa (Phaeophyceae) reveal that Asian specimens assigned to this taxon are genetically distinct. Botanica Marina 57(5): 351-358. DOI: https://doi. org/10.1515/bot-2014-0029

León-Álvarez, D., M. L. Núñez-Resendiz y M. E. Ponce-Márquez. 2014b. Morphological and molecular characterization of Neoralfsia hancockii comb. nov. (Ralfsiales, Phaeophyceae) from topotype of San José del Cabo, Baja California, Mexico. Botanica Marina 57(2): 139-146. DOI: https://doi. org/10.1515/bot-2013-0095

León-Álvarez, D., V. P. Reyes-Gómez, M. J. Wynne, M. E. PonceMárquez y N. Quiróz-González. 2017. Morphological and molecular characterization of Hapalospongidion gelatinosum, Hapalospongidiaceae fam. nov. (Ralfsiales, Phaeophyceae) from Mexico. Botanica Marina 60(5): 567581. DOI: https://doi.org/10.1515/bot-2017-0020

Lim, P. E., M. Sakaguchi, T. Hanyuda, K. Kogame, S. M. Phang y H. Kawai. 2007. Molecular phylogeny of crustose brown algae (Ralfsiales, Phaeophyceae) inferred from $r b c \mathrm{~L}$ sequences resulting in the proposal for Neoralfsiaceae fam. nov. Phycologia 46(4): 456-466.

Lozano-Orozco, J. G., A. Sentíes, F. F. Pedroche y J. Díaz-Larrea. 2016. Dictyota chalchicueyecanensis sp. nov. (Dictyotales, Phaeophyceae) en el Golfo de México: evidencias moleculares y morfológicas. Hidrobiológica 26(2): 225231. DOI: https://doi.org/10.24275/uam/izt/dcbs/ hidro/2016v26n2/lozano

Lozano-Orozco, J. G., A. Sentíes, J. Díaz-Larrea, F. F. Pedroche y O. de Clerck. 2014. The occurrence of Dictyota canariensis
(Dictyotales, Phaeophyceae) in the Gulf of Mexico. Botanica Marina 57(5): 359-365. DOI: https://doi.org/10.1515/bot2013-0111

Lozano-Orozco, J. G., A. Sentíes, O. de Clerck, K. M. Dreckmann y J. Díaz-Larrea. 2015. Two new species of the genus Dictyota (Phaeophyceae: Dictyotales) from the Mexican Caribbean. American Journal of Plant Sciences 6(15): 2492-2501. DOI: https://doi.org/10.4236/ajps.2015.615251

Luna Ortega, I. y V. de la Cruz-Francisco. 2017. Ensambles de macroalgas sobre superficies muertas de corales escleractinios (Anthozoa: Scleractinia) en el arrecife Oro Verde, Veracruz, México. CICIMAR Oceánides 32(1): 11-23. Mateo-Cid, L. E. y A. C. Mendoza-González. 1991. Algas marinas bénticas de la Isla Cozumel, Quintana Roo, México. Acta Botanica Mexicana 16: 57-87. DOI: https://doi. org/10.21829/abm16.1991.626

Mateo-Cid, L. E., A. C. Mendoza-González y C. Galicia García. 1996. Algas marinas de Isla Verde, Veracruz, México. Acta Botanica Mexicana 36: 59-75. DOI: https://doi. org/10.21829/abm36.1996.762

Mateo-Cid, L. E., A. C. Mendoza-González y R. B. Searles. 2002. New mexican records of marine algae including Crouania mayae sp. nov. (Ceramiaceae, Rhodophyta). Caribbean Journal of Science 38(3-4): 205-221.

Mateo-Cid, L. E., A. C. Mendoza-González y R. B. Searles. 2006. A checklist and seasonal account of the deepwater Rhodophyta around Cozumel Island on the Caribbean Coast of Mexico. Caribbean Journal of Science 42(1): 39-52.

Mateo-Cid, L. E., C. Mendoza-González y S. Fredericq. 2013 a. A checklist of subtidal seaweeds from Campeche banks, Mexico. Acta Botanica Venezuelica 36(2): 95-108.

Mateo-Cid, L. E., A. C. Mendoza-González, A. G. Ávila-Ortiz y S. Díaz Martínez. 2013b. Algas marinas bentónicas del litoral de Campeche, México. Acta Botanica Mexicana 104: 53-92. DOI: https://doi.org/10.21829/abm104.2013.57

Mateo-Cid, L. E., A. C. Mendoza-González y C. M. Hernández Casas. 2019. Diversity of brown algae (Ochrophyta, Phaeophyceae) of Sian Ka'an Reserve Biosphere, Mexican Caribbean. Pakistan Journal of Botany 51(1): 361-366. DOI: https://doi.org/10.30848/pjb2019-1(40)

Mattio, L. y C. E. Payri. 2009. Taxonomic revision of Sargassum species (Fucales, Phaeophyceae) from New Caledonia based on morphological and molecular analyses. Journal of 
Phycology 45(6): 1374-1388. DOI: https://doi.org/10.1111/ j.1529-8817.2009.00760.x

McDevit, D. C. y G. W. Saunders. 2017. A molecular investigation of Canadian Scytosiphonaceae (Phaeophyceae) including descriptions of Planosiphon gen. nov. and Scytosiphon promiscuus sp. nov. Botany 95(7): 653-671. DOI: https:// doi.org/10.1139/cjb-2017-0042

Mendoza-González, A. C. y L. E. Mateo-Cid. 2000. La familia Sphacelariaceae (Sphacelariales, Phaeophyta) en las costas de México. Polibotánica 11: 21-48.

Mendoza-González, A. C. y L. E. Mateo-Cid. 2005. El género Dictyopteris J.V. Lamouroux (Dictyotales, Phaeophyceae) en las costas de México. Hidrobiológica 15(1): 43-63.

Mendoza-González, A. C., L. E. Mateo-Cid y R. B. Searles. 2007. Yucatán seaweeds from the offshore waters of Isla Mujeres, Quintana Roo, Mexico. Botanica Marina 50(5): 280-287. DOI: https://doi.org/10.1515/bot.2007.032

Mendoza-González, A. C., L. E. Mateo-Cid y P. H. López Garrido. 2013. Algas marinas bentónicas asociadas a pecios y otras estructuras submareales de Campeche, México. Acta Botanica Venezuelica 36(2): 119-140.

Mendoza-González, A. C., L. E. Mateo-Cid y D. Y. García-López. 2017. Inventory of benthic marine and estuarine algae and Cyanobacteria for Tabasco, Mexico. Biota Neotropica 17(4): e20170379. DOI: https://doi.org/10.1590/1676-0611bn-2017-0379

Mendoza-González, C., L. E. Mateo-Cid, D. Y. García-López, J. A. Acosta-Calderón, A. Vázquez-Rodríguez, C. M. HernándezCasas, A. Gerardo y A. Garduño-Acosta. 2016. Marine seaweeds of the Yucatan Peninsula: Diversity, economic importance and conservation. In: Riosmena-Rodríguez, R. (ed.). Marine Benthos: Biology, Ecology Functions, and Environmental Impact. Nova Science Publishers Inc. New York, EUA. Pp. 83-138.

Nava-Olvera, R., L. E. Mateo-Cid, A. C. Mendoza-González y D. Y. García-López. 2017. Macroalgas, microalgas y cianobacterias epífitas del pasto marino Thalassia testudinum (Tracheophyta: Alismatales) en Veracruz y Quintana Roo, Atlántico mexicano. Revista de Biología Marina y Oceanografía 52(3): 429-439. DOI: https://doi. org/10.4067/s0718-19572017000300002

Ortega, M. M. 1995. Observaciones del fitobentos estuarino de la Laguna de Términos, Campeche, México. Anales del
Instituto de Biología, Universidad Nacional Autónoma de México, Serie Botánica 66(1): 1-36.

Ortega, M. M., J. L. Godínez Aguilar y G. Garduño-Solórzano. 2001.

Catálogo de algas bénticas de las costas mexicanas del Golfo de México y Mar Caribe. Instituto de Biología, Universidad Nacional Autónoma de México. Cd. Mx., México. 594 pp.

Ortegón-Aznar, I., J. González-González y A. Sentíes-Granados. 2001. Estudio ficoflorístico de la Laguna de Río Lagartos, Yucatán, México. Hidrobiológica 11(2): 97-104.

Ortegón-Aznar, I., I. Sánchez-Molina y R. H. Casanova Cetz. 2009. The distribution of marine algae in a coastal lagoon, northern Yucatan Peninsula, Mexico. Neotropical Biology and Conservation 4(2): 99-105. DOI: https://doi. org/10.4013/nbc.2009.42.05

Pedroche, F. F. y A. Sentíes. 2003. Ficología marina mexicana: Diversidad y Problemática actual. Hidrobiológica 13(1): 2332.

Pedroche, F. F. y A. Sentíes. 2020. Diversidad de macroalgas marinas en México. Una actualización florística y nomenclatural. Cymbella 6(1): 4-55.

Pedroche, F. F., A. Sentíes, E. Novelo y E. Meave Del Castillo. 2009. Algas. In: Ramírez Pulido, J. (ed.). Cosmos: Enciclopedia de las ciencias y la tecnología en México, Tomo II. Consejo Nacional de Ciencia y Tecnología/Universidad Autónoma Metropolitana. Cd. Mx., México. Pp. 55-69.

Pérez-Jiménez, G. M., M. G. Rivas-Acuña, D. León Álvarez, B. Campos Campos y N. Quiroz-González. 2020. Macroalgas de la laguna "El Carmen", Tabasco, México. Acta Botanica Mexicana 127: e1606. DOI: https://doi.org/10.21829/ abm127.2020.1606

Quan-Young, L. I., M. A. Díaz-Martín y J. Espinoza-Ávalos. 2006. Algas epífitas de Bajo Pepito, Isla Mujeres, Quintana Roo, México. Revista de Biología Tropical 54(2): 317-328. DOI: https://doi.org/10.15517/rbt.v54i2.13872

Ramírez Rodríguez, A. y R. Blanco Pérez. 2011. Macroalgas bentónicas marinas: conocimiento actual. La Biodiversidad en Veracruz, Estudio de Estado, vol. 2. Comisión Nacional para el Conocimiento y Uso de la Biodiversidad (CONABIO), Gobierno del Estado de Veracruz, Universidad Veracruzana, Instituto de Ecología, A.C. Xalapa, México. Pp. 51-57.

Robinson, N. M., C. Galicia-García y Y. B. Okolodkov. 2012. New records of green (Chlorophyta) and brown algae (Phaeophyceae) for Cabezo Reef, National Park Sistema 
Arrecifal Veracruzano, Gulf of Mexico. Acta Botanica Mexicana 101: 11-48. DOI: https://doi.org/10.21829/ abm101.2012.24

Sánchez-Molina, I., J. González-Ceballos, C. Zetina-Moguel y R. Casanova-Cetz. 2007. Análisis de la biodiversidad de algas marinas situadas entre Uaymitún y Chuburná, Yucatán. Ingeniería 11(1): 43-51.

Sánchez Rodríguez, M. E. 1980. Ficoflora del sustrato rocoso dentro de las costas del Golfo de México, México. Boletim do Instituto Paulista de Oceanografía 29(2): 347-350. DOI: https://doi.org/10.1590/s0373-55241980000200069

Sentíes, A. y K. M. Dreckmann. 2011. Reino Vegetal-Diversidad de especies: Macroalgas. In: Villalobos Zapata, A. (ed.). La biodiversidad en Campeche, Estudio de Estado. Comisión Nacional para el Uso de la Biodiversidad (CONABIO), Universidad Autónoma de Campeche, El Colegio de la Frontera Sur (ECOSUR). Cd. Mx., México. pp. 202-207.

Sentíes, A. y K. M. Dreckmann. 2013. Lista actualizada de las macroalgas de Tabasco, México. Acta Botanica Venezuelica 36(2): 109-117.

Tapia-Silva, F. O., O. E. Hernández-Cervantes, M. I. Vilchis, A. Sentíes y K. M. Dreckmann. 2015. Mapping of algae richness using spatial data interpolation. The International Archives of the Photogrammetry, Remote Sensing and Spatial Information Sciences XL-7/W3: 1005-1008. DOI: https:// doi.org/10.5194/isprsarchives-xl-7-w3-1005-2015
Thiers, B. 2021. Index Herbariorum: a global directory of public herbaria and associated staff. New York Botanical Garden's. Nueva York, EUA. http://www.nybg.org/bsci/ih/ih.html (consultado agosto de 2021).

Valadez-Cruz, F., G. Rosiles González y A. Ortega-Rubio. 2014. Diversidad de algas en la Reserva de la Biósfera Sian Ka'an, Quintana Roo. Investigación y Ciencia 22(60): 23-36.

Vilchis, M. I., K. M. Dreckmann, E. A. García-Trejo, O. E. Hernández y A. Sentíes. 2018. Patrones de distribución de las grandes macroalgas en el golfo de México y el Caribe mexicano: una contribución a la biología de la conservación. Revista Mexicana de Biodiversidad 89(1): 183-192. DOI: https://doi. org/10.22201/ib.20078706e.2018.1.2226

Wynne, M. J. y J. M. de Castro Nunes. 2021. The proposal of Rosenvingea endiviifolia (Martius) comb. nov. (Scytosiphonaceae, Phaeophyceae). Notulae Algarum 187: $1-4$. 